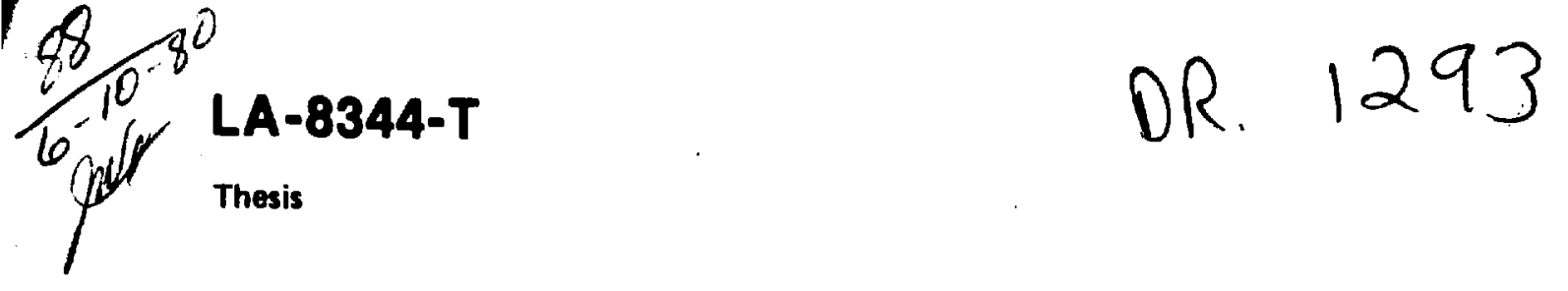

MASTER

\title{
Solar Wind Flows Associated with Hot Heavy lons
}


ILLUSTRATIONS............................... vi

ABSTRACT.................................... 1

I. INTRODUCTION............................... 2

II. INSTRUMENTATION AND DATA REDUCTION................ 4

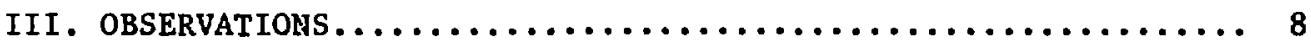

Range of Spectra observed....................... 8

Solar Wind Characteristics Associated with High

Ionization States.......................... 13

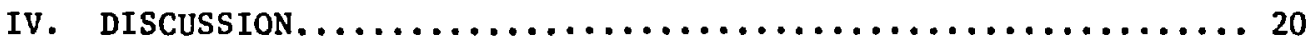

Solar Origins for Some NCDEs..................... 20

Classes of Flows Associated with Hot Heavy Ions........... 21

Active Regions as Sources of the Low Speed Solar Wind....... 25

v. COMPARISON WITH MODELS....................... 27

vi. concLusions.............................. 36

APPENDIX A: DATA REDUCTION...................... 38

B: $\chi^{2}$ FITS TO MODELS....................... 41

The January 3, 1975, Event.............. 41

The March 5, 1973, Event............... 46

ACKNOWLEDGMENTS.............................. 50

REFERENCES.................................. 51 


\section{ILLUSTRATIONS}

\section{Figure}

1 Two examples of hot heavy ion spectra with NCDEs....... 6

2 Range of ionization states observed................ 9

3 Contour map of a scatter plot of the solar wind temperature and velocity for the periods studied....... 22

4 Heuristic models of the solar wind expansion with hot heavy ions.............................. 24

5 Expansion, Ionization, and recombination time scales.... 30

6 Ionization balance from equation 3 and from the local temperature.............................. 32

$7 \quad x^{2}$ surfaces for the January 3, 1975, spectrum........ 44

8 Modified $x^{2}$ surfaces for the March 5, 1973, spectrum.... 48 
SOLAR WIND FLOWS ASSOCIATED WITH

HOT HEAVY IONS

by

Edward Eugene Fenimore

\begin{abstract}
Solar wind heavy ion spectra measured with the Vela instrumentation have been studied with the goal of determining the solar origins of various solar wind structures which contain anomalously high ionization states. Since the ionization states "freeze-in" close to the sun they are good indicators of the plasma conditions in the low and intermediate corona. Heavy ion spectra from three different periods throughout the solar cycle have been analyzed. These data are consistent with freezing-in temperatures ranging from $21.5 \times 10^{6} \mathrm{~K}$ to higher than $9 \times 10^{6}$. The spectra indicating hot coronal conditions occur in roughly $1 / 7$ of all measurements and almost exclusively in postshock flows (PSFs), nonshock related helium abundance enhancements (HAEs), or noncompressive density enhancements (NCDEs). The PSFs and HAEs are both probably interplanetary manifestations of solar flares. The observation of several flare-related HAEs which were not preceded by an interplanetary shock suggests that the flareheated plasma can evolve into the solar wind without producing a noticeable shock at $1 \mathrm{AU}$. The NCDEs with hot heavy ions differ from the PSF-HAEs in several ways implying that they evolve from events or places with lower temperatures and less energy than those associated with the flares, but with higher temperatures and densities than the quiet corona. Active regions, coronal mass ejections, and equatorial streamers are possible sources for the NCDEs with spectra indicating hot coronal conditions. These events owe their enhanced densities to coronal processes as opposed to interplanetary dynamical processes. High ionization temperatures are observed almost always in either the NCDEs or the flare-related PSF-HAEs; therefore, if a nonflaring active region evolves into the solar wind with hot heavy ions, it probably evolves into an NCDE. Since NCDEs make up less than $10 \%$ of the solar wind, it is suggested that the slow speed solar wind rarely originates in the high temperature portion of active regions. Models of the solar wind expansion demonstrate how some NCDEs can have extreme, nonequilibrium ionization distributions.
\end{abstract}




\section{INTRODUCTION}

The observation of solar win: ions heavier than helium (Bame et al., 1968) can be a useful tool in studying the relationship between the solar corona and the solar wind. The relative abundance of different ionic states of an element in the solar wind is determined by the temperature, density, and expansion characteristics of the -olar corona inside $5 R_{0}$ (Hundhausen, Gilbert, and Bame, 1968). Thus, measurements of the ionic states of the solar wind by in situ satellites at $1 \mathrm{AU}$ can provide information on the conditions at the solar

wind base which is unaffected by the passage of the plasma through interplanetary space. This "freezing-in" of the lonic states is in contrast to most other parameters of the solar wind (density, speed, temperature) which are modified in interplanetary space and, in general, bear few unambiguous signatures of the base plasma conditions.

Solar wind heavy ions have been observed using electrostatic analyzers and the deployment of metal folls on the moon. The electrostatic analyzers have indicated the presence of various ionic states of oxygen, silicon, and iron with probable contributions from nitrogen, sulfur, and other less abundant elements (Bame et al., 1970, 1975; Holzer and Axford, 1970; Lange and Scherb, 1970). In addition, the metal foll experiments have yielded the solar wind abundance by isotope for the inert gases neon and argon (see e.g., Geiss et al., 1970). Previous studies of the ionization balance of the heavy ions measured with the Vela electrostatic analyzers (Bame et al., 1968, 1974, 1978) have identified two broad types of heavy ion spectra occurring in the low to moderate speed solar wind. The first is observed within the interstream 
plasma which comprises most low speed, low temperature, flows at $1 \mathrm{AU}$. The coronal temperature gradients consistent with the heavy ion spectra for this type of flow agree well with the value expected for a heat conduction dominated, spherically symmetric corona: $a=2 / 7$ (Bame et al., 1974). The temperature structure was assumed to be of the form

$$
T=T_{0}\left(\frac{r}{R_{0}}\right)^{-\alpha}
$$

where $T_{0}$ is the temperature at the surface, that is at 1 solar radius, $R_{0}$. Much less frequently, spectra are observed which can be self-consistently interpreted as evolving from a hotter than normal corona (Bame et al., 1978 ). These high ionization states, combined with the fact that in some cases the spectra occur in the abnormally depressed kinetic temperature plasma following interplanetary, flare-related shocks, form the basis for associating these hot heavy ion spectra with flare ejecta (Bame et al., 1978). Two other examples of hot heavy lons have been reported (Bame et al, 1973). A careful check of all available solar and solar wind data showed that these two examples were not flare related.

It is the purpose of this paper to investigate the types of solar wind flows which are associated with hot heavy ions, 1.e. heavy ion spectra that can be shown to have evolved from regions with unusually high ionization temperatures. The three major conclusions are: (1) hot heavy ions occur almost exclusively in noncompressive density enhancements (NCDEs, Belcher and Davis, 1971; Gosling et al., 1977), helium enhancenents, or post-shock flows; (2) at least some of the solar wind flows labeled NCDE owe their enhanced densities to the sun rather than interplanetary dynamical processes; and (3) very little 10-- speed, low temperature solar wind evolves from a hotter than normal corona. 


\section{INSTRUMENTATION AND DATA REDUCTION}

The heavy ion observations were made with the Los Alamos Scientific Laboratory's (LASL) hemispherical electrostatic analyzers on the four Vela 5 and 6 earth-oriented satellites. Previous studies (Bame et al., 1968, 1970, 1974, 1975; Feldman et al., 1974) utilized the Vela data from 1969 to 1971. Details of the orbit and data accumulation method can be found in Bame et al. (1970) and Bame (1972). For the purpose of this paper only those details affecting t: e observations will be presented. Basically, the energy per charge (E/Q) range of 1 to $8 \mathrm{kV}$ is measured in four different segments (each with 20 intervals) over an 8.5 minute period of time. Within this time two spectra (labeled A and B spectra) are accumulated, each with 80 energy-per-charge samples of constant $\Delta E / E$. The B spectra differ from the $A$ spectra in that the sensor threshold is raised, thereby discriminating against lighter ions. Such discrimination assists in verifying that the observations are indeed heayy lons and not bow shock reflected protons (e.g., Asbridge, Bame, and Strong, 1968). Since the efficiency for the A spectra is $\sim 100 \%$, this paper will display only the A spectra.

Sets of spectra were summed together into combined spectra with improved statistics. The combined spectra were shifted so as to position the He ${ }^{+2}$ peak at $2 \mathrm{kV}$. This shift, combined with the self-consistent result that all heavy ions normally flow at about the same speed as the protons and helium (Bame et al., 1968), results in each ion having its peak at the kV position numerically equal to its mass per unit charge (M/Q). In the following, therefore, the $E / Q$ 
spectrum will often be referred to as an M/Q spectrum. Appendix A gives additional details of the analysis procedure.

Figure 1 presents two typical combined spectra. Here, the $10 \mathrm{~g}$ of the counts per $\mathrm{kV}$ (arbitrary normalization) is plotted against the $10 \mathrm{~g}$ of $\mathrm{M} / \mathrm{Q}$. Beneath the $\mathrm{He}^{+2}$ peak are brackets indicating the Vela energy interval width, the RMS jitter of the speed during the measurement and the maximum shift in velocity between two successive spectra. The small brackets beneath the curve (e.8., at $\sim 2.5,4.5$, and $7.5 \mathrm{M} / \mathrm{Q}$ in the January 3,1975 spectra) are the approximate locations and sizes of the gaps between the four segments of the spectrum. Bulk speed variations will tend to fill in the gaps between tire segments (see Appendix A). The brackets above the curves specify $E / Q$ ranges which will be compared to models of the expansion in $\mathrm{V}$.

Vertical lines have been used in figure 1 to indicate the positions of the more abundant ionic states of oxygen, silicon, and iron. Several other ions can have an important contribution to the spectrum. In particular there can be fairly large contributions due to $s^{+9}, s^{+9}$, and $s^{+10}$ at $M / Q$ values near those of $\mathrm{Si}^{+7}, \mathrm{Si}^{+8}$, and $\mathrm{Si}^{+9}$ respectively. In addition, $\mathrm{Mg}^{+10}$ and $\mathrm{Si}^{+12}$ often account for $20 \%$ of the counts near the $0^{+7}$ position. More complete lists of the locations of possible contributors can be found in Holzer and Axford (1970), Lange and Scherb (1970), and Bame et al. (1972). The vertical lines in figure 1 are intended only to guide the reader in identifying peaks and are not intended to indicate that other ions have negligible contributions.

The above discussion demonstrates the key uncertainty involved in the data. Several different elements can contribute to the same value of $M / Q$. In order to estimate the reezing-in temperatures whlle the abundances are not completely known, we must rely on self-consistent arguments based on expected ranges of elemental abundances and coronal temperatures (Bame, 1972; Bame et 


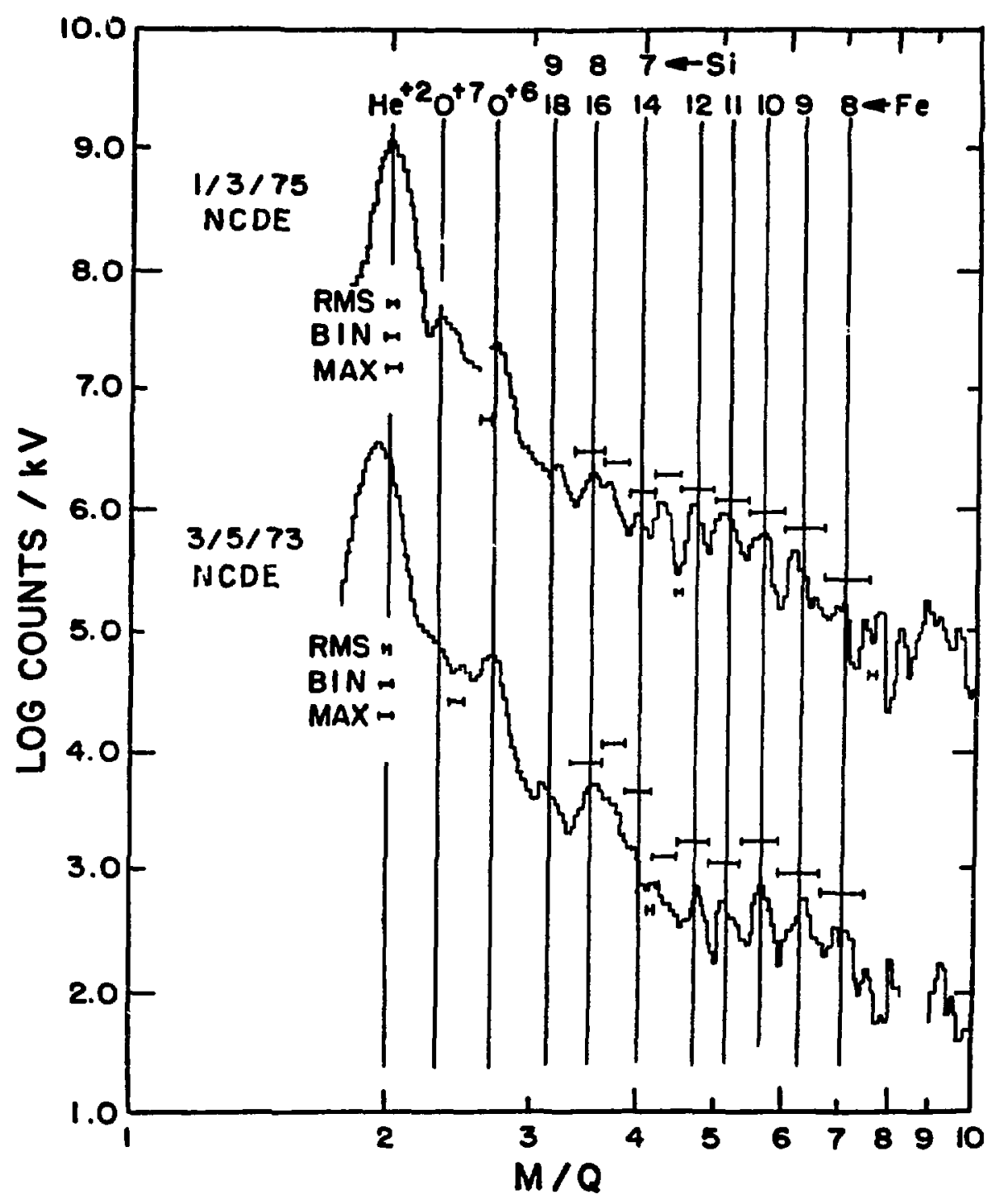

Fig. 1. Two examples of hot heavy ion spectra associated with NCDEs. 
al.; 1979, III of this paper) or quantitative models (Bame et al., 1974;

V). The uncertainty in the abundances will not change the qualitative conclusions to be presented. 
III. OBSERVATIONS

\section{a) Range of Spectra Observed}

The Vela satellite system has provided heavy ion observations from June 1969 to the present. Three periods of time representative of the solar cycle were selected for analysis. These periods were: February 1971 to August 1971, February 1973 to August 1973, and November 1974 to May 1975. Since the conclusions of this paper were found to be valid in all three periods, the three intervals were combined into a single set of observations. Individual spectra were accumulated into a combined spectrum until there was some indication of either a change in the freezing-in temperature or a change in the character of the solar wind flow. From the three periods under consideration, 340 combined spectra were obtained on 164 different days. On the average, the combined spectra contained $\sim 8$ individual spectra representing about one hour of observing time. Many of the comblned spectra were discarded because their high kinetic temperatures blurrec the peaks together making a determination of the ionization temperature nearly impossible. This left 156 spectra during 88 different intervals of time (i.e., "events"). This large data base reveals the wide range and nearly continuous gradation of heavy ion distributions which occur in the solar wind.

Selected heavy ion spectra covering the full range of observed freezing-in temperatures are plotted in Fig. 2. Top to bottom in the left panel and then top to bottom in the right panel follows a sequence from the coolest to hottest spectra observed so far. The spectra in Fig. 2 will be combined with 


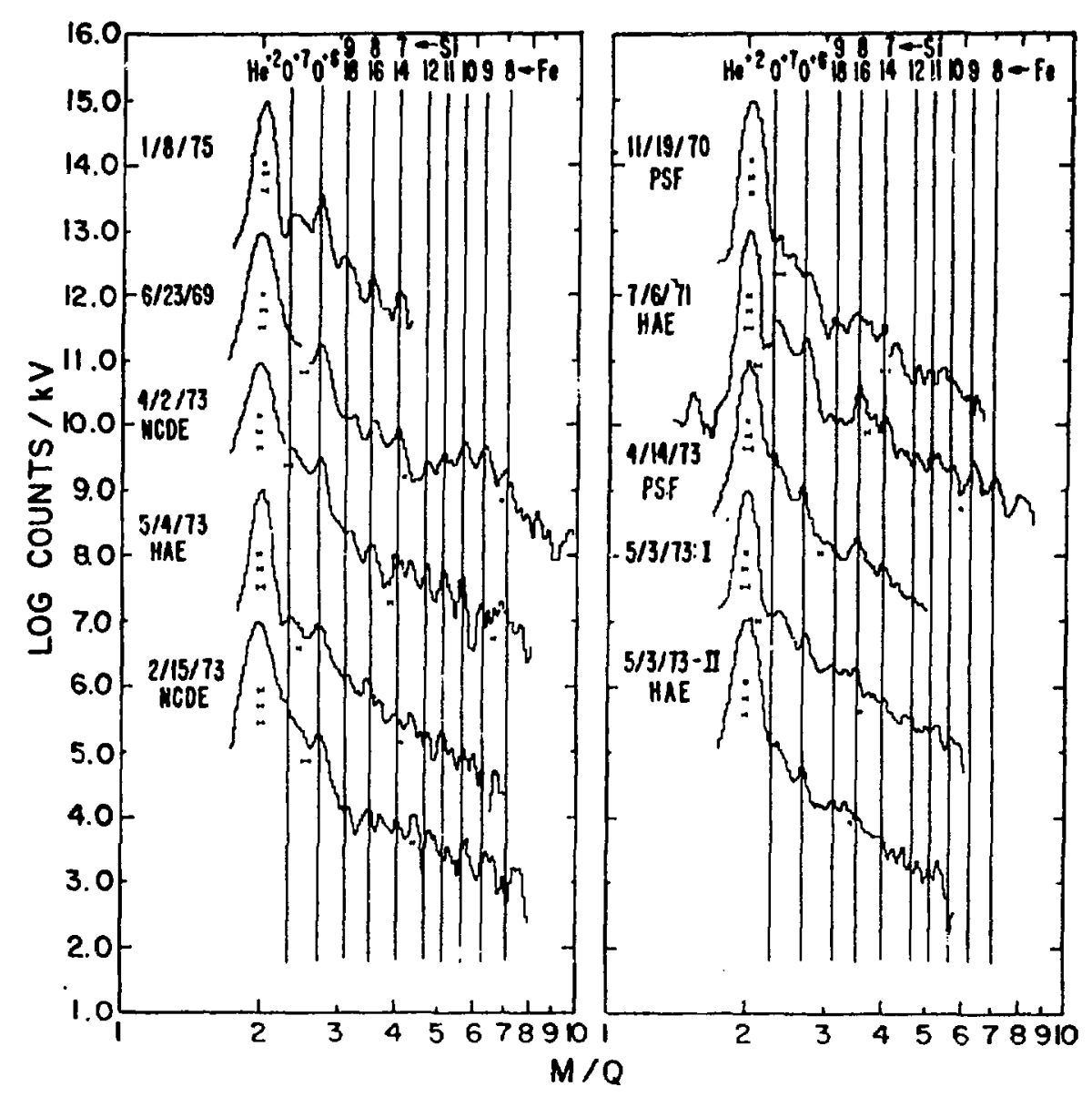

Fig. 2. Top to bottom in the left panel and then top to bottom in the right panel follows a sequence from the coolest to hottest ionization states observed so far in the solar wind. 
self-consistent arguments similar to (or extensions of) those used by Bame et al. (1968, 1979) in order to demonstrate how the heavy ion spectra can be classified as either normal, hot, or intermediate.

The dividing lines between normal, hot, and intermediate are somewhat subjective. In general the spectra designated normal will have the $M / Q=2.29$ peak (predominantly $0^{+7}$ ) height approximately equal to that of $M / Q=2.67$ (predominantly $0^{+6}$ ), the three peaks normally seen between M/Qs of 3.0 and 4.0 (imedominantly $\mathrm{Si}^{+7}, \mathrm{Si}^{+8}$, and $\mathrm{Si}^{+9}$ ) will have decreasing amplicude with increasing $M / Q$, and the envelope of peaks between $M / Q$ of 4.0 and 8.0 (predominantly $\mathrm{Fe}^{+14}$ to $\mathrm{Fe}^{+7}$ ) will have a maximum at about $\mathrm{M} / \mathrm{Q}$ equal to 6.0 . The June 23, 1969 spectrum is a classical example of such a normal spectrum. This spectrum has been studied in detail in the past (Bame et al., 1974, 1975, Feldman et al., 1974). Tha above features have been shown to indicate that the coronal temperature at the height for the freezing in of the oxygen ionization states $\left(\Omega \cdot 1.5 \mathrm{R}_{0}\right)$ was $2.1 \times 10^{6}{ }^{\circ} \mathrm{K}$ and was $1.58 \times 10^{6}{ }^{\circ} \mathrm{K}$ at about 3 $R_{0}$ where the iron freezes in. Spectra similar to that of June 23, 1969 are designated normal becaise the majority of low-speec, interstream solar wind flows have similar spectra.

The characteristics of hot spectra have been presented by Bame et al. (1979). The kev differences between the hot and normal spectra are that the peak at 2.29 is larger than that at 2.67 by at least a factor of $\backsim 1.6$, the peak at $M / Q$ equal to 3.5 is enhanced relative to the peaks at 3.1 and 4.0 (presumably due to $\mathrm{Fe}^{+16}$ ), and the envelope of peaks between 4.0 and 8.0 is greatly reduced in relative intensity. The two post-shock flow spectra in figure 2 (November 19, 1970 and April 14, 1973) are excellent examples of hot spectra. In studying these two spectra as well as other post-shock flows, Bane et al. (1979) showed that the ratio of the 2.29 and 2.67 peaks implied a 
freezing-in temperature for oxygen of about $3.4 \times 10^{6} \mathrm{~K}$ and that the peak at $3.5 \mathrm{M} / \mathrm{Q}$ was predominately $\mathrm{Fe}^{+16}$ impl ing a freezing-in temperature for iron of about $2.9 \times 10^{6} \% \mathrm{~K}$.

In addition to the examples described above and those in previous studies, figure 2 demoristrates that there are intermediate cases. Usually an intermediate spectrum will have one or two of the characteristics of a hot spectrum although perhaps not to the same degree. For example, in the April 2, 1973 spectrum, the envelope of peaks between $M / Q s$ of 4.0 and 8.0 does not have a maximum near $M / Q \sim 6.0$ and thus, is at a higher tempe ature than the June 23 , 1969 spectrum but at a lower temperature than the September 19, 1970 spectrum. The April 2, 1973 spectrum is approximately the (subjective) dividing line between normal and intermediate.

The May 4, 1973 spectrum is another example of an intermediate spectrum. Although the peak at $3.5\left(\mathrm{Fe}^{+16}\right.$ and/or $\mathrm{Si}^{+8}$ and $\left.\mathrm{s}^{+9}\right)$ is enhanced relative to the peak at $4.0\left(\mathrm{Si}^{+9}\right.$ and/or $\left.\mathrm{Fe}^{+14}\right)$ and $\mathrm{Fe}^{+15}$ is present, the $\mathrm{O}^{+7}$ to $\mathrm{o}^{+6}$ ratio is approximately unity and the feaks beyond $M / Q$ equal to 4.0 are not reduced in intensity to the same degree as in the hot spectra. Based on arguments similar to Bame et al. (1968, 1979), it appears that both the oxygen and iron freezing-in temperatures were $\sim 2 \times 10^{6} \mathrm{~K}$ which (if they froze-in at the normally assumed, widely separated positions) implies an isothermal atmosphere (and therefore heating) above $1.5 \mathrm{R}_{0}$. It is also possible that the various ion species all froze in at the same coronal altitude due to a very steep density gradient or a sudden acceleration.

The hottest spectra observed so far occurred on May 3, 1973. The May 3, 1973-I spectrum (fig. 2) shows all the standard characteristics of a hot spectrum. In addition, there are two peaks as intense as the $\mathrm{M} / \mathrm{Q}=3.5$ peak (predominantly $\mathrm{Fe}^{+16}$ ) at $\mathrm{MQ}=3.3$ and 3.1 which are the expected locations 
for $\mathrm{Fe}^{+17}$ and $\mathrm{Fe}^{+18}$ respectively. The $\mathrm{Fe}^{+17}$ peak is a particularly convincing Indicator ce a very hot spectrum because it is at a location in $M / Q$ which is not contaminated by other elements if the temperature is higher than $\sim 2 x$ $10^{6 \circ} \mathrm{K}$. The $\mathrm{W} / \mathrm{Q}$ peaks at 2.29 and 2.67 imply a freezing-in temperature for $\mathrm{O}^{+7}, \mathrm{O}^{+6}, \mathrm{Si}^{+12}, \mathrm{Mg}^{+9}$, etc. of $\sim 2.6 \times 10^{60} \mathrm{~K}$ but $\mathrm{Fe}^{+16}, \mathrm{Fe}^{+17}$, and $\mathrm{Fe}^{+18}$ are approximately equally abundant at a temperature of $1.4 \times 10^{7 \circ} \mathrm{K}$. This implies a much higher temperature at the freezing-in level of iron than at the level for oxygen, silicon, etc. The May 3, 1973 II spectrum is at an even higher temperature. The $M / Q=2.29$ to 2.67 ratio inplies a temperature of $\backsim 3.2 \times$ $10^{60} \mathrm{~K}$. The $\mathrm{Fe}^{+17}$ peak is now more intense than the $\mathrm{Fe}^{+16}$ peak implying a temperature of $\sim 1.6 \times 10^{70} \mathrm{~K}$.

It is not expected that steep density gradients or sudden accelerations could make the iron states freeze in at lower altitudes than the elements responsible for the 2.29 and 2.67 peals. Since the May 3-I spectrun lasted for a time ( $3.25 \mathrm{hr}$.$) long compared to the replenishment time for the corona$ (10 min.), explanations should consider the corona to be in a steady state. A possible explanation $1 \mathrm{~s}$ that the coronal temperature maximum was $21.4 \times 10^{70} \mathrm{~K}$ and was between the freezing in levels of the elements of the 2.29 peak (oxygen, silicon, etc.) and that of iron. If correct, then some form of coronal heating must have taken place at very high levels. In alternate explanation is that a mixture of plasmas was observed with the $\mathrm{Fe}^{+17}$ due to one component with a temperature of $\sim 1.6 \times 10^{7}$ and the elements in the $\mathrm{M} / \mathrm{Q}=$ 2.29 and 2.67 peaks due to different components with a lower temperature.

We note that temperatures in excess of $1.0 \times 10^{70} \mathrm{~K}$ quoted above are based on recombination and Ionization coefficients determined from Sumers $(1974 a, b)$. The variations between the different calculations of coefficients can cause different values to be assigned to the freezing-in tenperature. For 12 
example, if the Jordan (1968 and private communication) coefficients were used, the May 3 spectra would have lower temperatures assigned to them (n 9.0 $x 10^{6} \mathrm{~K}$ ). However, employing different coefficients would not change the order of the spectra in figure 2 or the qualitative conclusion of this paper; rather it affects the absclute freezing-in temperatures assigned to the spectra.

At times the freezing-in temperature becomes ambiguous because of poor statistics, large thermal broadening of the peaks, large bulk velocity shifts, or the presence of peaks at unusual $M / Q$ locations. The ambiguous spectra coulc be mixtures of plasmas which originated at two or more different tenperatures or perhaps from a normal cemperature structure but with unusual abundances. To avoid blasing the statistics describing the classification, the statistics will be presented twice; with and without the spectra with ambiguous interpretation.

\section{b) Solar Wind Characteristics Associated with High Ionization States}

In order to determine when solar regions hotter than nomal are the source of the low speed solar wind, we will investigate what types of solar wind flows are associated with hot heavy ions. For instrumental reasons (Bame, 1970) our observations do not cover all types of solar wind flows. This fact is illustrated in Figure 3 where a contour map summarizes the average hourly proton temperature and velocity values of the solar wind for the periods studied. The broken line very roughly divides the temperature-velocity space into the region where the heavy ions are observed to be resolved and the region where the kinetic temperature blurs the peaks together (i.e., above the 1ine). Nearly all of the 156 spectra used to associate flows with hot heavy 
lons fall below this line. The conclusions drawn in this paper are, therefore, only valid for those flows beneath the line.

An overview of solar wind flow types observed to acconpany the measured heavy ion spectra showed that they can be segregated into one of four categories: 1) post-shock flows (PSPs), 2) helium abundance enhancenents (HAEs, Hirshberg et al., 1970, 1972), 3) noncompressive density enhancements (NCDEs, Belcher and Davis, 1971; Gosling et al., 1977), or 4) interstrean. Table 1 presents the distribution of normal, intermediate, and hot heavy ion spectra among the 4 defined types of solar wind flows. The c assification was based on the LASL IMP 6,7 , and 8 and the Vela 5 and 6 plasma parameters. The "unknown" entry refers to heavy ion spectra for which there were no IMP or Vela plasma parameters available. Column A differs from column B in that column B includes those spectra for which estimates of the freezing-in temperatures were ambiguous. There are two salient features of Table 1. First, the hot spectra occur almost exclusively in NCDEs, HAEs, and PSFs. Only 1 spectrum out of 21 was ionizationally not while not being identified with a NCDE, HAE, or a PSF. The second salient feature is that normal ionization temperatures are seen in all types of flows. It is not likely that this is solely an effect of incomplete sampling of the events. In many cases we had virtually continuous coverage of entire NCDE and HAE events and still did not observe any hot heavy lons.

\section{1) Post-Shock Flows (PSFs)}

The solar wind flows following some interplanetarv shooks have been interpreled as resulting from solar flares (Hirshberg et al., 1970, 1972). These PSFs are often marked by an enhanced heljum abundance (Hirshberg et al., 1970), abnormally low proton kinetic tenperatures (Gosling et al., 1973), 
TABLE 1

Number of Heavy Ion Spectra For Various Solar wind Flows

\begin{tabular}{|c|c|c|c|c|c|c|}
\hline \multirow[b]{2}{*}{ FLOW TYPE } & \multicolumn{2}{|c|}{$\begin{array}{c}\text { NORMAL } \\
\text { FREEZING IN } \\
\text { TEMPERATURES }\end{array}$} & \multicolumn{2}{|c|}{$\begin{array}{l}\text { INTERMEDIATE } \\
\text { FREEZING IN } \\
\text { TEMPERATURES }\end{array}$} & \multicolumn{2}{|c|}{$\begin{array}{c}\text { HOT } \\
\text { FREEZING IN } \\
\text { TEMPERATURES }\end{array}$} \\
\hline & A & $\mathrm{B}^{1}$ & $A$ & B & $\mathbf{A}$ & B \\
\hline Interstream & $34(25)^{2}$ & $36(26)$ & $5(5)$ & $8(6)$ & $1(1)$ & $1(1)$ \\
\hline NCDE & $16(10)$ & $19(10)$ & $8(5)$ & $9(6)$ & $6(5)$ & $9(7)$ \\
\hline HAE & $5(4)$ & $5(4)$ & $5(4)$ & $6(5)$ & $5(2)$ & $6(3)$ \\
\hline Post-Shock Flows & $8(2)$ & $8(2)$ & NONE & NONE & $5(3)$ & $5(3)$ \\
\hline Unknown & $37(11)$ & $42(13)$ & $2(2)$ & $2(2)$ & NONE & NONE \\
\hline
\end{tabular}

1 The B colimen includes ambjguous spectra.

2 The number in parentheses is the number of events. 
and/or an abnormally low electron kinetic temperature (Montgomery et al., 1974). It is reasonable to expect that the driver gas (see e.g., Hundhausen, 1972) was heated by the flare and has high ionization states. Indeed, the heavy ions in the driver gas for some PSFs have been shown to be anomalously hot as reported separately by Bame et al., 1979. Those PSFs with hot heavy ions are marked by circles in figure 3.

\section{1) Helium Abundance Enhancement (HAEs)}

Although helium enhancements are a common feature of PSFs, not all helium enhancements are associated with solar flares and interplanetary shocks (Hirshberg et al., 1972.). We wish to distinguish between heavy ion spectra Identified as the flare heated driver gas and other heavy ion spectra which are also characterized by an unusual hellum abundance. We therefore cleine flow conditions to be a HAE whenever the one-hour average of the helium fraction is above $9 \%$, but excluding those times which are Identified as the driver gas for a shock. Since only $\backsim 3 \$$ of the observations have helium fractions greater than 9\$ (Robbins, Hundhausen, and Bame, 1970) the arbitrary dividing line of $9 \$$ insures that few spectra will be mistakenly designated as an HAE.

The observation of hot heavy ions within HAEs can link the HAE with a particular event on the sun. For example, on July 6, 1971 the IMP 6 satellite measured the helium fraction to be as high as $15 \%$. The average velocity was $400 \mathrm{~km} / \mathrm{sec}$ with a kinetic temperature of $\sim 10^{40} \mathrm{~K}$. Although the ion temperature during the event was anomalously low for the speed, a shock was not observed or inferred from the occurrence of a geomagnetic sudden commencement. The heavy ion spectrum observed during the 23rd hour of July 6 , 1971 most certainly indicates a high ionization temperature (see Fig. 2). It 
is reasonable to identify the origin of the HAE plasma with a $1 \mathrm{~B}$ flare which occurred at $18 \mathrm{~h} 46 \mathrm{~min}$. on July 2, 1971. The basis for this association is the lack of other flares during the interval of interest and the fact that the time difference between the flare and the observation corresponds to a solar wind speed of $414 \mathrm{~km} / \mathrm{sec}$ in agreement with the observed $400 \mathrm{~km} / \mathrm{sec}$. Either 1) the shook had dissipated, 2) it caused a variation too small to notice at 1 $A U$, or 3) the $1 B$ flare (with no type II or IV radio burst) did not produce a shock. The probable identification of a flare with the July 6, 1971 HAE suggests that flare ejecta can evolve into the solar wind without producing a noticeable shock at $1 \mathrm{AU}$.

During the May 3, 1973 event ( $\mathrm{fig} .2$ and $\mathrm{IV}-\mathrm{a}$ ), the helium fraction was often above $10 \%$ and ranged up to $20 \%$. These spectra (shich had the highest ionization states observed so far) can be identified with a series of flares, the largest being a 2B flare at $5 \mathrm{~h} 50$ min on April 30. The transit time to 1 AU implied by this association yields an average velocity of $\sim 508 \mathrm{~km} / \mathrm{sec}$ which compares favorably with the range of velocities observed during the heavy ion spectra $(495$ to $520 \mathrm{~km} / \mathrm{sec})$. However these spectra, although within a temperature depression, did not have an interplanetary shock or sudden commencement assoclated with them. The nearest sudden commencement occurred $s$ 48 hours before the May 3 spectra. (No interplanetary shock at the time of the sudden commencement was observed due to a data gaj.) The transit time leads to associating the sudden commencement with a series of 1B or 1N flares which started at $3 \mathrm{~h} 51 \mathrm{~min}$ on April 29. We speculate that the $1 \mathrm{~B}$ flares on April 29 produced an unobserved shock and formed a solar wind disturbance similar to those previously proposed for flare disturbed solar wind (e.g., Hundhausen, 1972). The driver gas for the shock probably arrived at 1 AU at $s$ 
$12 \mathrm{~h}$ on Nay 2. Subsequently the 2B flare of April 30 fed flare heated material into the solar wind which was observed as hot heavy ions on May 3; however, no new shock is noticeable. The effect of the April 30 flares was to prolong the disturbance at $1 \mathrm{AU}$ for up to 72 hours.

\section{iii) Noncompressive Density Enhancements (NCDEs)}

NCDEs have been discussed by Belcher and Dav1s (1971) and defined quant1tatively by Gosling et al. (1977). They are solar wind flows characterized by an unusually high density not readily associated with an interplanetary compressicn. Briefly, an event is designated an NCDE if the density is above

$15 \mathrm{~cm}^{-3}$ and the speed and temperature are either constant or decreasing with time.

Explanations for NCDEs fall into two categories. In one category the NCDEs are thought to evolve from dense coronal regions. For example, it has been suggested that slow-moving coronal mass ejections (CMEs) are the source of some NCDEs (Gosling et al., 1977). Alternatively, the filaments of dense slow-speed flow evolving from equatorial streamers or active regions might be responsible for some of the NCDEs. The other category of possible explanations does not involve coronal density enhanced plasma. Rather, interplanetary dynamical processes may be involved so that the apparent NCDEs are really compressive (Dryer, Steinolfson, and Wu, 1976). For example, Pizzo (1976) has calculated three-dimensional models of the solar wind expansion which give compressive density enhancements that are not accompanied by immediate Increases in radial velocity. Evidence that at least some NCDEs are not directly related to plasma conditions involving solar activity is the fact that the occurrence rate for NCDEs is the same at solar minimum and maximum (Feldman et al., 1978). 
An inspection of Table 1 shows that only a minority of the NCDE events with unambiguous Ionization temperatures contained hot heavy ions. of 20 events, hal $f$ had normal ionizational temperatures and one quarter had hot freezing-in temperatures.

\section{iv) Other Flows}

Out of 21 spectra with possibly hot heavy ions, only 1 was not associated with an NCDE, HAE, or PSF. The exception occurred on February 21, 1973. The February 21 spectrum is represented by the cross in Figure 3 . It has the

largest thermal temperature $\left(\backsim 5.6 \times 10^{4} \mathrm{~K}\right)$ of the hot spectra and occurred in a compression in the solar wind. Although above the line whlch roughly marks the limit of the instruments' ability to resolve the heavy ion peaks, the February 21 spectrum had a statistically significant enhancement of the peak at $M / Q=3.5\left(\mathrm{Fe}^{+16}\right)$ and was therefore classified as hot. It is possible that this event was, in reality, an NCDE which was overtaken by higher velocity plasma and converted into a compression. In fact, if we were making our observations at a greater distance from the sun (e.g., $5 \mathrm{AU}$ ), we would expect that almost all of the NCDEs would be compressed by the evolving stream structure. 


\section{DISCUSSION}

\section{a) Solar origins for some HCDEs}

As desoribed above (s III-b-iii) there are conflicting explanations for the source of the density enhancements in NCDEs: coronal vs interplanetary. The heavy ion observations can help distinguish between some of these possible explanations. If rot heavy ions are observed in a NCDE, then the NCDE must be associated with some solar hot region. The explanation that the NCDEs involve normal density plasma heated by a solar hot region and then (coincidently) compressed to a high density by interplanetary dynamical processes is untenable because if that were the situation, one would expect to see examples of hot heavy ions that are not associated with NCDEs. The interstream solar wind rarely (if ever) contains hot heavy ions. Thus when hot heavy ions are observed in a NCDE, it is reasonable to deduce that the density enhancement occurred in the corona where the ionization states are set. We conclude that NCDEs with hot heavy ions are manifestations of coronal density enhancements associated with a solar hot region. The fact that the occurrence rate for NCDEs is the same at solar minimum as it is at solar maximum (Feldman et al., 1978) implies that solar activity can not explain all NCDEs. of the 10 NCDE events with normal freezing-in temperatures (see Table 1), some may be due to interplanetary dynamical processes and some to coronal density enhancements which do not involve high ionization states. 


\section{b) Classes of flows associated with hot heavy lons}

Next we sho; that actually there exist probably just two classes of flows associated with hot heavy ions: flare related flows which are manifested in PSFs or HAEs and a distinct class, manifested as NCDEs.

The HAE events have many characteristics which are almost identical to the PSFs studied by Bame et al. (1979) except no noticeable shocks are observed. The July 6, 1971 and May 3, 1973 events (discussed in 5 III-b-1i) were the onlv HAEs associated with heavy ions which were unambiguously hot. Both events had very clear associations with flares. Although the sample is small we believe that this implies at all HaEs with hot heavy ions are flare related. The NCDEs with hot heavy ions appear to be different from the PSF-HAEs in frur ways.

1) The PSF-HAEs with hot heavy ions ure all in anomalously cool solar wind and the NCDEs are mostly above the demarcation line between anomalously cool and normal solar wind (see Fig. 3).

2) The NCDEs in general have a lower ionizational temperature than the PSF-HAEs. The freezing in temperature for iron in NCDEs range from the (subjective) lower 1 imit for hot spectra $\left(n 2.3 \times 10^{6} \circ \mathrm{K}\right)$ to $\sim 2.8 \times 10^{6} \mathrm{~K}$. In constrast HAEs and PSFs have freezing-in temperatures for iron which range up to $1.6 \times 10^{7}{ }^{\circ} \mathrm{K}$ (see Figs. 1 and 2 ).

3) The NCDEs often have a wider range of ionizational states than the PSF-HAEs. In the PSFs the envelope of iron states can be roughly fit by an isothermal ionization balance (Bame et al., 1979). However, in 3 of the 5 NCDEs with hot heavy lons, a range of ionization states was observed which probably can not be fit by either an isothermal ionization balance or the ionization balance obtained using the coronal characteristics 


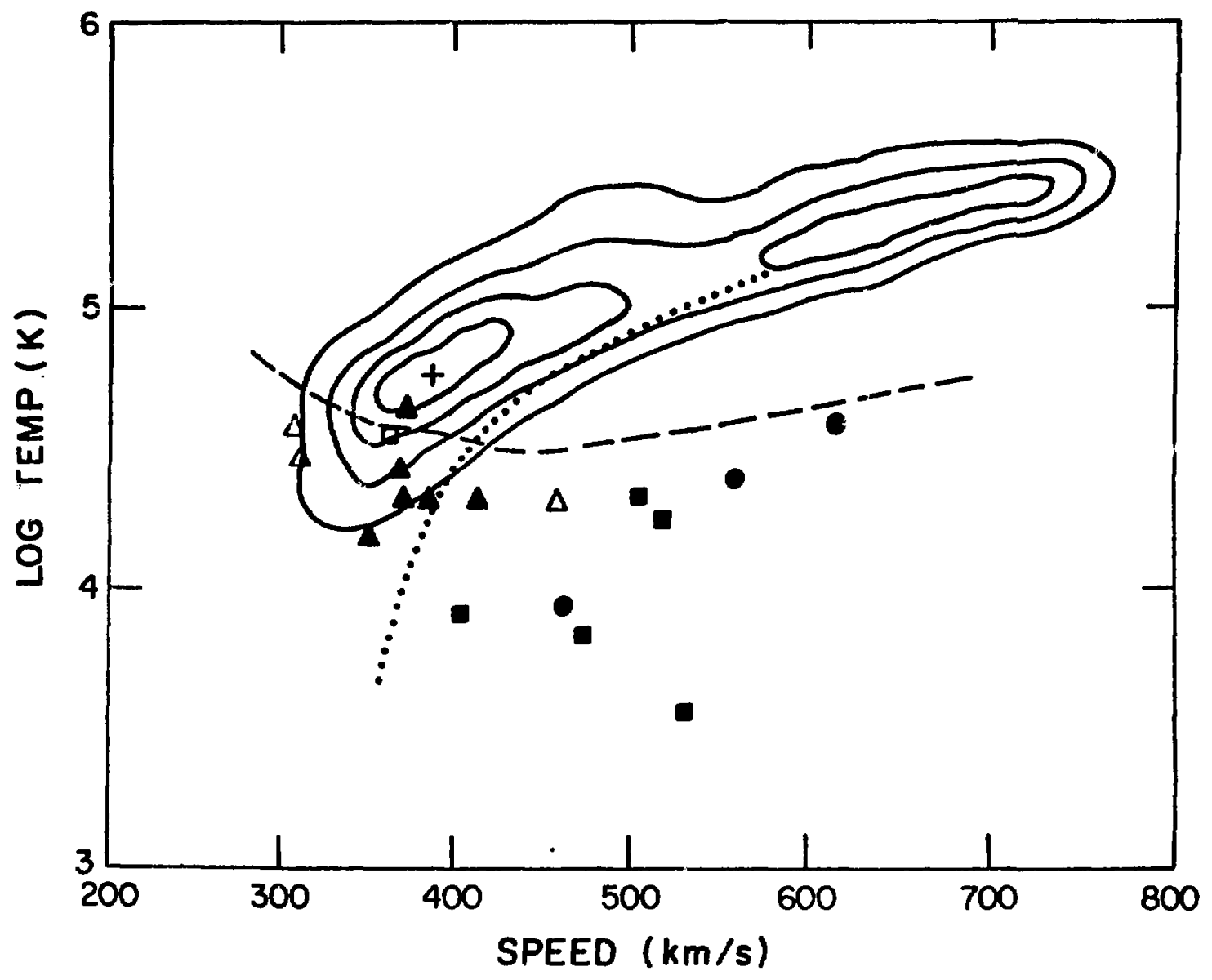

Fig. 3. Contour map of a scatter plot of the solar wind temperature and velocity for the periods studied. The broken line roughly divides the region of good instrumental resolution (beneath) from poor resolution. The dotted line demarks anomalously cool solar wind from normal (from Gosling et al., 1973). The circles, squares, and triangles are respectively PSFs, HAEs, and NCDEs with hot heavy ions. Open symbols have ambiguous temperature interpretations. 
assumed by Bame et al. (1974). This will be treated in greater detail in s v.

4) The final distinction between the NCDEs and PSF-HAEs is that most NCDEs are not flare-related, whereas all of the PSFs and HAEs with hot heavy ions had very clear associations with flares. Of the 7 NCDE events which possibly contained hot heavy ions, only 2 could possibly be associated with flares.

These differences can be combined into the heuristic model shown in Figure 4 which is a view from above the solar pole looking down onto the ecliptic plane. The effects of solar rotation have been ignored. The Pr T-HAEs with hot heavy ions are thought, to result from large flares which can heat the plasma to high ionization states and provide the energy for a greater than $r^{-2}$ areal expansion (the flare model in Fig. $4 \mathrm{a}$ is basically taken from Hundhausen, 1972). The larger than $r^{-2}$ expansion cools the plasma to an anomalously low kinetic temperature. (It is also possible that the magnetic field forms a bubble or bottle which inhibits interplanetary heating, see Gosling et al., 1973 and Montgomery et al., 1974). The observed nearly isothermal ionizational distributions in PSF-HAEs are consistent with a rapid initial expansion.

In contrast, the NCDEs with hot heavy ions are postulated to result from less energetic events, active regions, or equatorial streamers which do not result in ionization states as high as those seen in the PSF-HAE spectra. The smaller energy released is usually not enough to provide the expansion characteristics which would produce anomalously cool solar wind at 1 AU (see Fig. 4a). The NCDEs are slightly cooler (on the average) than the rest of the solar wind suggesting that perhaps the expansion for the NCDEs is slightly faster than $r^{-2}$ but not nearly as fast as in the PSF-HAEs. Figure $4 \mathrm{~b}$ elaborates on these points by showing two possibilities for the origin of the NCDEs 


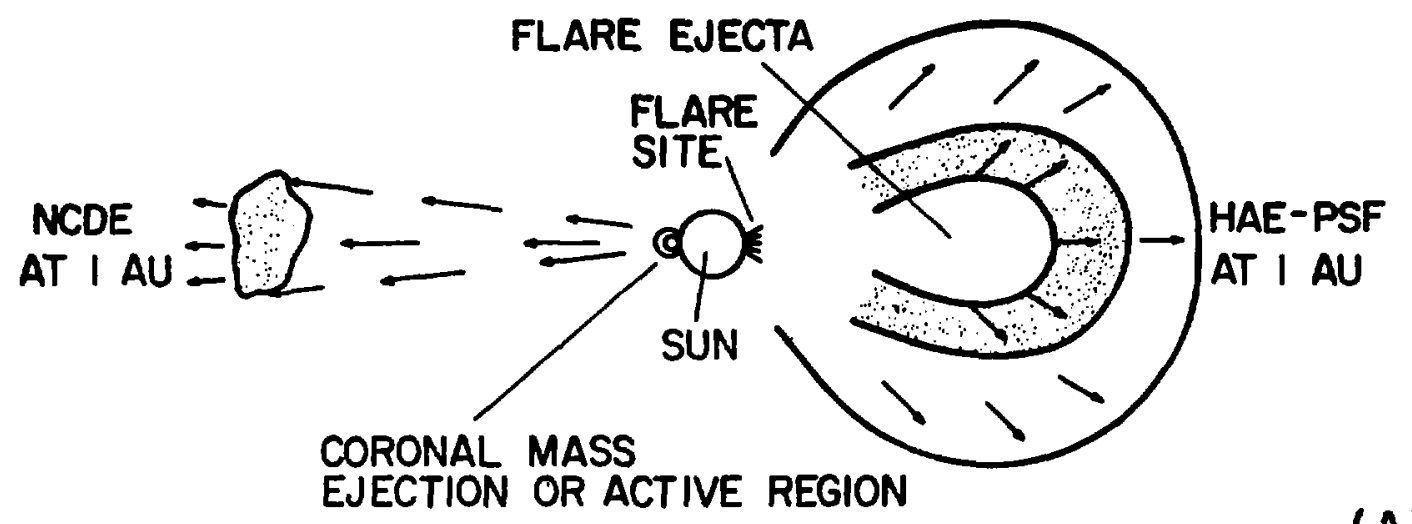

(A)
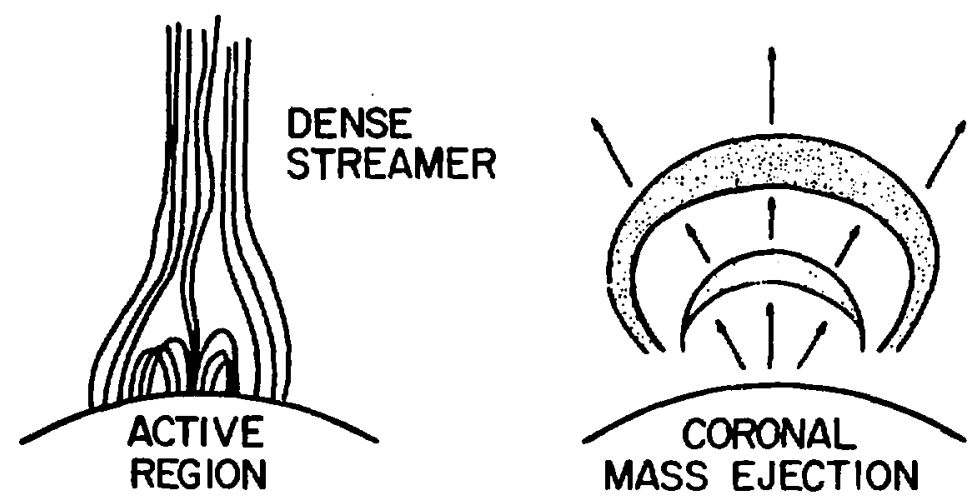

(B)

Fig. 4. Heuristic models of the solar wind expansion with hot heavy ions. (a) The PSF-HAEs probably involve an expansion greater than $\mathrm{r}^{-2}$ to provide the anomalously cool solar wind. (i) The sources of NCDEs with hot heavy ions probably involve active region streamers, or coronal mass ejection because they have the requisite high coronal density, high temperature, yet probably do not expand as much as the flare disturbances. 
with hot heavy lons. The slow moving coronal mass ejections (CMEs, see e.g., MacQueen et al., 1974) involve events which release less energy than does a flare, yet they provide the requisite coronal density enhancement. Streamers over active regions also provide the requisite density and coronal temperature enhancements. Equatorial streamers (e.B., Newkirk, 1967) are an alternative possibility. Models of streamers have shown temperature and density enhancements at the altitudes where heavy ions freeze-in (e.g., Pneuman, 1973). The equatorial streamers are often seen at solar minimum which might help explain the fact that the occurrence rate for NCDEs is the same at solar minimum and solar maximum (Feldman et al., 1978).

\section{c) Active regions as sources of the low speed solar wind}

The sources of the low speed, low temperature solar wind are presently not known. The fact that hot heavy ions occur almost exclusively in PSFs, HAEs or NCDEs can put limits on the sources of the low speed, low temperature solar wind. It is fairly well established that the sources of the PSF-HAEs are flares and that they occur in the anomalously low temperature, high speed plasma (1.e., to the right of the dotted line in Fig. 3). Thus hot heavy ions are observed in the low speed solar wind only in connection with a NCDE. Therefore, if plasma occasionally evolves from a solar hot region into the low speed solar wind, our observations indicate that such plasma probably evolves only into a NCDE. This conclusion assumes normal expansion times for the plasma; a slow diffusion of plasma from a hot region might allow the high Ionization state to recombine before freezing-in.

Our observations are consistent with the suggestion that one source of the slow speed solar wind is active regions. However, we believe those active regions evolve only into NCDEs, are infrequent, and probably evolve along open 
fleld lines. In addition to the above discussion linking nonflaring solar hot regions with only NCDEs, three other considerations support this opinion. 1) Calculated models of the coronal magnetic field (e.g., Levine, 1978) connect open field lines in some active regions to solar wind speed minima and that is where NCDEs are typically seen. 2) Such open field lines should provide the requisite conditions for having hot heavy lons in the solar wind: normal (i.e., similar to those summarlzed by Newkirk, 1967) expansion velocities and a locally high value of the electron temperature. 3) A dense streamer over the active region can provide the density enhancement which is eventually manifested as a NCDE at $1 \mathrm{AU}$.

The observations summarized in Table 1 can put an upper limit on the fraction of the slow speed solar wind which evolves from active regions via open field lines. This fraction is probably less than 58 because 1) roughly 68 of the nonflare-related heavy ion spectra (i.e., not PSF-HAEs) contain hot heavy ions (see Table 1), 2). NCDEs account for less than 10 of the solar wind (Gosling et al., 1977) and only $1 / 4$ of the NCDEs are observed to have hot heavy ions, and 3) there are probably NCDEs with hot heavy ions whose sources are not active regions, in particular we expect CMEs to explain some of the NCDES . 


\section{COMPARISONS WITH MODELS}

Our motivation for comparing the heavy ion observations within NCDE structures with detailed models of the corona is twofold: 1) to make quantitative estimates of freezing-in temperatures for tyoical NCDEs and 2) to propose one possible qualitative explanation of the extreme, nonequilibrium ionization distributions seen in some NCDEs. An example of such a distribution is seen in the March 5, 1973 spectrum (Fig. 1). Here significant $\mathrm{Fe}^{+16}$ and $\mathrm{Fe}^{+15}$ as well as significant $\mathrm{Fe}^{+10}$ and $\mathrm{Fe}^{+9}$ are simultaneously present. If an isothermal ionization balance is used, the $\mathrm{Fe}^{+10}$ and $\mathrm{Fe}^{+9}$ peaks are too big (relative to the $\mathrm{Fe}^{+16}$ ) by about a factor of 5.

In order to compare the heavy ion observations with models of the corona, a calculation of the solar wind heavy element ionization balance including the dynamical effects of the coronal expansion must be made. The relative abundances of the ionization states of a particular element are found as solutions of (Hundhausen et al., 1968)

$$
\vec{\nabla} \cdot\left(n_{i} \vec{u}\right)=n_{e} n_{i-1} c_{i-1}-n_{e} n_{i}\left(C_{i}+R_{i}\right)+n_{e} n_{i+1} R_{i+1} .
$$

where $n_{i}$ is the relative abundance of the ith ionization state (i.e. with a charge of +1$), n_{e}$ is electron density, $\vec{u}$ is the velocity vector, $C_{i}(T)$ is the collisional ionization coefficient for transitions from ionization state $i$ to $i+1$ (as a function of temperature), and $R_{i}(T)$ is the recombination coefficient for transitions from state 1 to $1-1$. The parameters $n_{e}, n_{1}, u$, and $T$ are 
functions of the position $r$ in the corona and equation (2) is solved to find the $n_{1}$ at $r=r_{E}$ where $r_{E}$ is $1 A U$. In practice the $n_{1}$ freeze-in at values of $r$ less than $\& 10 R_{0}$. Therefore, the calculation was only carried out as far as ir $\sim 24 R_{0}$.

If mass conservation and spherical symmetry are assumed, equation (2) reduces to the one-dimensional equation (Bame et al., 1974)

$$
\frac{d\left(n_{i} / n_{e}\right)}{d \ln r}=\frac{n_{e} r^{3}}{F}\left[n_{1-1} c_{1-1}-n_{1}\left(c_{1}+R_{1}\right)+n_{1+1} R_{1+1}\right]
$$

where $F$ is a constant equal to the mass flux $\left(n_{e} V r^{2}\right)$ with $V$ being the solar wind bulk speed.

In order to solve equation (3) only the density and temperature structure need to be specifled. In all our models, we will assume the temperature structure is given by equation (1). The density structure will be one of two different models. The first is a modification of the Newkirk (1967) density model to include a multiplicative scaling parameter $(\gamma)$. The purpose of the $\gamma$ parameter is to determine the effect of steady state coronal density enhancements.

The purpose of the second density model is to demonstrate how variations from the Newkirk standard model ant explain the observed, extreme, nonequilibrium ionization distributions. The second density model was roughly patterned after CMEs because CMEs have been suggested as possible sources for some NCDEs (Gosling et al., 1977). Unfortunately, there are very few quantitative observations of the density and velocity profiles for CMEs. In addition, some simplifications are necessary to keep the solution of equation (3) computationally feasible. The following considerations are the basis for our CME model. The typical loop structure of a CME (MacQueen et al., 1974; Gosling et al., 1974) is often fit better by symmetry from the surface rather than symmetry from the center of the sun. Symmetry from the surface would be 28 
expected if that is where the energy release occurs. Some CMEs are observed to expand away from the sun with near constant velocity. Indeed, the CME for which density and velocity profiles are available (the September 14-15, 1973 event, see Dulk et al, 1974) showed that the leading edge of the event was expanding at a constant speed. The speed of the material behind the leading edge is not directly observable. However, figure 3 of Dulk et al. shows no decrease or increase in density was produced behind the leading edge. Thus the material behind the leading edge was neither catching up nor falling behind. This implies that all of the material was, indeed, moving with roughly constant speed. The simplification of constant speed and symmetry from the surface results in a density profile of the form

$$
n_{e}=\frac{6 \times 10^{6}}{\left(\frac{r}{r_{0}}-1\right)^{2}}
$$

where the constant $6 \times 10^{6}$ was chosen because such a function (plus an additive constant of $1.25 \times 10^{5}$ ) fit very well the density profile reported by Dulk et al. Certainly some CMEs will ha: a some charceteristics which differ from the September 14-15 event. However, we are mainly interested in whether or not expansion characteristics which are different from the Newkirk model can qualitatively explain the observed nonequilibrium lonization distributions. In the model calculations, equation (4) will be modified by a scaling factor ( $\gamma$ ) to investigate the effects of varying the density.

Figure 5 demonstrates the differences in the freezing-in process between the Newkirk model and the CME model. Figure 5a shows the classical freezing-in explanation (Hundhausen et al., 1968). The expansion time scale, 


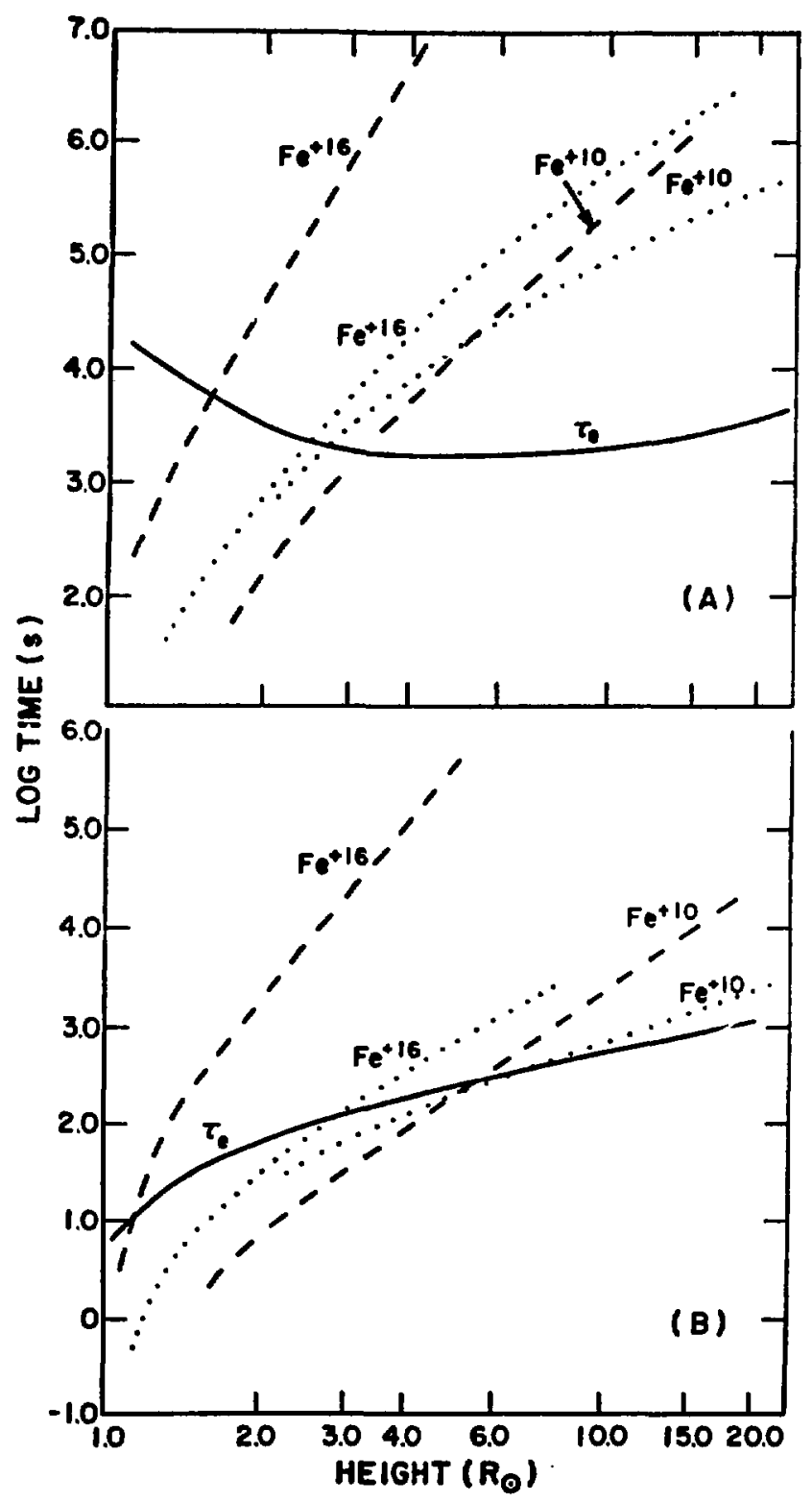

Fig. 5. Expansion, ionization, and recombination time scales (solid, broken, and dotted lines, respectively) for $\mathrm{Fe}^{+16}$ and $\mathrm{Fe}^{+10}$ (a) Newkirk model, (b) CME model. This demonstrates how the CME model can produce extreme, nonequilibrium tonization distributions. 
$\tau_{e}$, is $\left(V d\left(\ln n_{e}\right) / d r\right)^{-1}$ which is decreasing when $r \leqslant 6 R_{0}$. The time scales for ionization $\left(\tau_{I}^{-1}=n_{e} C_{1}\right)$ and recombination $\left(\tau_{R}^{-1}=n_{e} R_{1}\right)$ are increasing. Eventually the ionization balance is frozen because there is insufficient time within one scale height for ionization and recombination. The distribution deviates slightly from equilibrium because various ions freeze in at different heights where there are different electron temperatures. In Figure $5 a, T_{0}$ and a were respectively $4.9 \times 10^{6}$ and 0.4 .

In the case of the CME model, the assumption of constant velocity, mass conservation, and spherical symmetry from the surface gives $\tau_{e}=(r-$ $\left.R_{0}\right) /(2 V)$. This expansion time scale is plotted in Figure $5 \mathrm{~b}$ along with the ionization and recombination time scales. for $\mathrm{Fe}^{+16}$ and $\mathrm{Fe}^{+10}$. The same $\mathrm{T}_{0}$ and a as in Figure 5 a were used. Notice that the expansion time scale is now increasing with height and tends to spread out the freezing-in locations. The result is a more nonequilibrium distribution than with the Newkirk model. Since the ionization and recombination time scales are determined from the temperature and density profile, $\tau_{I}$ and $\tau_{R}$ are similar in both the Newkirk and CME model. The CME time scales are an order of magnitude smaller only because the density in the CME is roughly an order of magnitude larger than the Newkirk model.

Figure 6 shows the results of solving equation 3 for the density of $\mathrm{Fe}^{+16}$ and $\mathrm{Fe}^{+10}$ using the conditions assumed in figure 5. Figure 6a corresponds to the Newkirk model and figure $6 \mathrm{~b}$ is for the CME model. The solid lines are the solutions from equation 3 and the broken lines are the ionization balance determined from the local temperature as a function of height. Notice how the equation 3 solutions become constant (i.e., frozen-in) whereas the ionization balance due to the local temperature keeps changing. Whereas in the Newkirk model, $\mathrm{Fe}^{+16}$ and $\mathrm{Fe}^{+10}$ both freeze-in at $\sim 3.5 \mathrm{R}_{0}$, in the CME model, they 


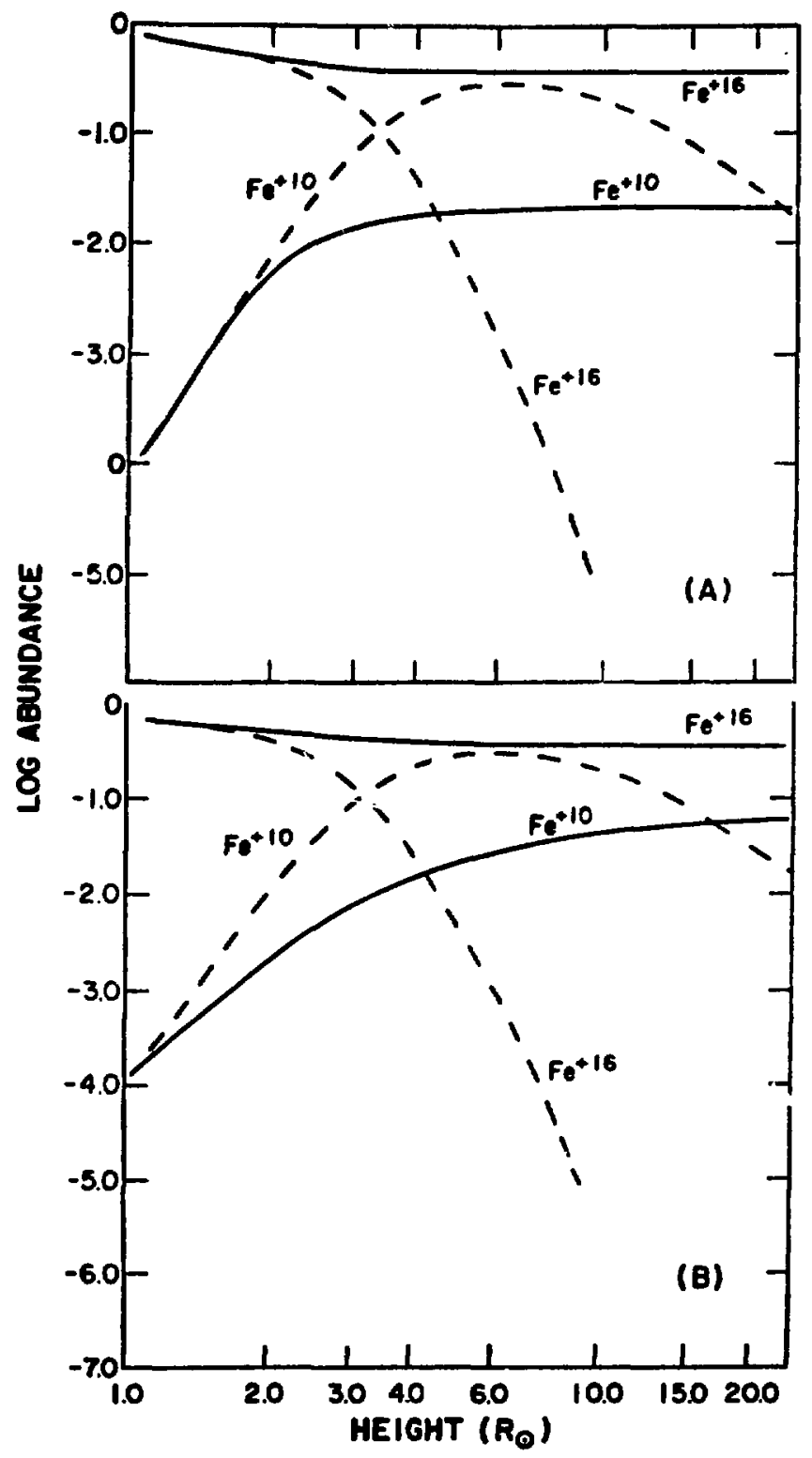

Fig. 6. Ionization balance from equation 3 (solid lines) and from the local temperature (broken lines) (a) Newkirk mode1, (b) CME model. 
freeze-in at $\backsim 3.5 \mathrm{R}_{0}$ and $10.0 \mathrm{R}_{0}$ respectively. As a result, the ratio of $\mathrm{Fe}^{+16}$ to $\mathrm{Fe}^{+10}$ is much less (a factor of 3 in Fig. 6) for the CME models than for the Newkirk model. Thus, the CME model can produce ionization states that are more out of equilibrium than can the Newkirk model. If $\alpha$ is chosen to be larger than 0.4, ever: more extreme, nonequilibrium distributions can be produced.

The key feature of the CME model which causes ionization distributions which are more nonequilibrium than those of the Newkirk model is the fact that the expansion time in the CME model is increasing with height rathel than decreasing. Note in Figure $5 \mathrm{a}$ that for distances greater than $\sim 7 R_{0}$, the Newkirk expansion time also becomes an increasing rather than a decreasing function. The reason for this is that eventually the Newkirk model also gives a constant velocity with spherical symmetry which causes $t$ to be equal to $r /(2 v)$. However, by the time the Newkirk model gives an increasing function, all the ions are already frozen in.

The above conclusions do not rely heavily on our basic assumptions. For the Newkirk models, the assumed form of the temperature profile (Eq. 1) is not crucial because the ions freeze-in over a short range of altitudes. Thus, the temperature profile at higher and lower altitudes have little influence over the observations. In the case of the CME models, the form of the temperature profile has more of an influence because the lons freeze-in gradually. Unfortunately, there are presently no observations of the temperature structure of a CME to provide an alternative temperature profile. We have also assumed a steady state for the corona. Although most of the events we are studying are intrinsically nonsteady state, the observed spectra remained effectively unchanged throughout each summation period (on the average about one hour). Thus the events were effectively in steady state during the period 
of time of the observations. Concerning the differences between the Newkirk model and the CME models, similar results would be expected for a variety of density and velocity profiles other than symmetry from thre surface and constant velocity. Slight accelerations or deaccelerations (as observed in many CMEs) or nonspherically symmetric flows could still produce the extreme nnnequilibrium ionization balance as long as $\tau_{e}=\left|\mathrm{Vd}\left(\operatorname{ll}_{\mathrm{n}} \mathrm{N}_{e}\right) / \mathrm{dr}\right|^{-1}$ is increasing.

The two spectra shown in Figure 1 were fit with 'the two different models to determine whether they were consistent, with the sstandard Newkirk model or CEM-like coronal conditions. The parameters $a, T_{0}$, and $\gamma$ were varied in order to model various temperature and density distributions. The velocity profile was determined by mass conservation. Appendix B gives additional details of the fitting procedure and results. Both events were NCDEs with hot heavy ions.

The January 3, 1975 event could be fit by a Newkirk model with $a=0.3, T_{0}$ $=3.4 \times 10^{6}$, and $y=1.0$. The $x^{2}$ with the best fit parameters was 8.28 with 5 degrees of freedom. The significance of this fit is twofold. 1) The $\gamma=1.0$ implies a coronal density enhancement was not necessary to obtain the ionization balance observed for the iron states. However, density enhancements by $a$ factor of $s 4$ are easily within the 10 confidence levels for the models. 2) The $\alpha=0.3$ is the same result found for the interstream spectra (Bame et al., 1974) and is consistent with a heat conduction dominated corona. In this context, the only difference between the interstream models and the Newkirk model for the January 3 event is a higher $T_{0}$. The CME model could also fit the January 3 event equally well ( $x^{2}$ only $5 \$$ larger than the Newkirk model). The best fit CME model had $\gamma=1.0$ which corresponds to the same density 
structure as seen in the September 14-15, 1973 CME (Dulk et al,, 1976). Thus, a density enhanced corona is also consistent with the January 3 NCDE data. In constrast to the January 3 event, the NCDE on March 5, 1973 had an extreme, nonequilibrium ionization distribution (see Fig. 1 and Appendix B). The CME model provided a better fit to the envelope of observed peaks than did the Newkirk model. Not only was the fit better, but the best fit Newkirk model required a very large a ( $>1.2$ ) which is difficult to justify if the atmosphere is heat conduction dominated. (The CME model had $\alpha \sim 0.43$ and $T_{0} \sim$ $4.3 \times 10^{6 \circ} \mathrm{K}$; close to the assumed conditions of Figs. 5 and 6.) The better fit of the CME model confirms that it has the general expansion characteristios required to help explain the nonequilibrium distribution. Whereas the Newkirk model must have a large a such that iron states freeze-in at largely different temperatures within a small range of distances, the CME model has the iron states freeze-in over a wide range of distances. The wider range of distances allows the iron states to respond to a wider range of temperatures without a large a. 


\section{vI. CONCLUSIONS}

Self-consistent arguments and models which account for the observed distribution of heavy ions can provide estimates of the temperature of the corona from which the solar wind evolved. The ionization temperature, combined with the observed solar wind flow, can be used to obtain several conclusions concerning the temperature structure of the corona and the manner in which the corona evolves into the slow speed solar wind.

1) In addition to the normal heavy ion spectra associated with interstream solar wind (Bame et al., 1968) and hot heavy lons identified in PSFs as the flare-heated driver gas (Bame et al., 1979), we find a continuous gradation of heavy ion spectra with ionizational temperatures ranging from $1.5 \times 10^{6}$ to $1.6 \times 10^{7} \mathrm{~K}$. The hot heavy fons occur almost exclusively in either PSFs, HAEs, or NCDEs. The HAEs are probably the same class of events as the PSF (i.e., flare-related) except no shock is observed. The association of an ionizationally hot HAE with a flare directly demonstrated that sometimes flare heated plasma can evolve into the solar wind without causing a noticeable shock at $1 \mathrm{AU}$.

2) NCDEs with hot heavy Ions are probably a different class of events than the PSF-HAEs. This conclusion rests on the facts that a) the NCDEs with hot heavy ions typlcally do not occur in anomalously cool solar wind, b) these NCDEs usually have lower fonizational temperatures than the HAE-PSFs, and c) these NCDEs often have an extreme, nonequilibrium ionizational distribution not seen as strongly in the PSF-HAEs. 
3) The density enhancement in $t$ NCDEs with hot heavy ions is due to a coronal density enhancement rather than due to interplanetary dynamical processes such as suggested by Dryer et al. (1976) and three dimensional models of the solar wind expansion (Pizzo, 1976). However these models may apply to those NCDEs with normal heavy ion spectra.

4) Table 1 and Fig. 3 have shown that hot nonflare related heavy ions occur in the low speed solar wind only in coinection with a NCDE. Therefore, plasma evolving from a solar hot region (such as an active region) into the solar wind probably evolves only into a NCDE. Because NCDEs account for less than $10 \%$ of the mass flux flowing past $1 \mathrm{AU}$ and only a fraction of them contain hot heavy ions, it is concluded that active regions and other hot coronal regions contribute only a minor amount to the low speed solar wind.

5) Coronal mass ejections, streamers over active regions, and equatorial streamers are suggested as the sources of the solar wind in NCDEs with hot heavy ions because those structures have the requisite coronal density erihancements and high temperatures. However, this leaves uncertain the sources of the majority of NCDEs which do not have hot heavy ions. Probably some are due to interplanetary dynamical process and some evolve from a denser-than-normal corona with a normal temperature. 


\section{APPENDIX A: DATA REDUCTION}

There are several orbital features of the Vela 5 and 6 sateliftes which limit the number of heavy ion spectra which can be analyzed (Bame, 1972). Collectively those effects eliminate 998 of the observing time. Besides the orbit related restrictions, interpretation of the spectra is difficult or impossible in certain types of solar wind flows. If the kinetic temperature

of the piasma protons is much above $3 \times 10^{40} \mathrm{~K}$, the peaks from the individual ionization states are so wide in the $E / Q$ spectra that identification of the peaks and subsequent estimation of their heights become very difficult. Most solar wind flows at speeds greater than $400 \mathrm{~km} / \mathrm{sec}$ will have kinetic temperatures which preclude useful heavy ion spectra. Taken together, the orbit-related restrictions and the flow-related restrictions have limited the useful observations to a small fraction of one percent of the available time. We have modified the data reduction technique (e.g., Bame et al., 1974, Feldman et al., 1974) in order to lift some of the restrictions imposed upon previous studies of the Vela heavy ion data base. Roughly an order of magnitude more spectra were thusly obtained. To sum the individual spectra into a combined spectrum including the effects of shifts in velocity, a fixed set of energy intervals was established. The counts in the measured intervals were mapped into the fixed intervals under the assumption they were uniformly distributed. The fixed set then contains the same counts per $\mathrm{kV}$ as in the observed spectrum. As the solar wind speed varies, the position of an ionic peak in the heavy ion spectrum will vary (position $=1 / 2 M V^{2} / Q$ where $M / Q$ is 
the heavy ion mass per unit charge and $V$ is the common bulk speed). Effectively, the varying speed causes each individual spectrum to sanple the true spectrum at slightly different $M / Q$ positions. Thus, five individual spectra of 80 energy per charge intervals each at different bulk speed means that the true spectrum was sampled with 400 different interval centers. In order to take advantage of this effect, the number of fixed intervals was three times the number of measured intervals.

The technique of using a larger number of fixed bins than true intervals has beneficial and detrimental effects on the data reduction. The two main beneficial effects are that the shifting spectra will fill in the small gaps between the four segments of 20 bins and that an optimum resolution can be achieved. However, the four different segments are collected at $\backsim 2$ minute intervals, and, yet, the required shift is obtainable only from the segment which contains the $\mathrm{He}^{+2}$ peak. Thus if the speed is varying on a time scale of $\sim 4$ minutes, the speed determined from the segment with the helium peak could cause the position of the iron and silicon peaks to vary from their actual positions. This would tend to increase the width of individual peaks. In order to estimate this effect, the RMS of the velocity jitter was calculated and is presented with the reduced spectra under the He ${ }^{+}$peak (see Fig. 1). Since the energy intervals of the Vela analyzers were logarithmically spaced, $\Delta E / E$ is a constant and the interval sizes shown at the He+ ${ }^{2}$ peak are typical for the whole spectrum. Note that the spectra are histograms with each bar equal in size to one third of a Vela energy interval.

We illustrate our reduction and presentation procedure with the January 3 , 1975 spectrum (see Fig. 1). Here, the solar wind speed was $\sim 342 \mathrm{~km} / \mathrm{sec}$ and the helium peak was shifted from $1.241 \mathrm{kV}$ to $2 \mathrm{kV}$ for presentation purposes. The roughly 1 to $8 \mathrm{kV}$ spectrum was mapped into a set of fixed intervals 
covering the range of 1.55 to $12.4 \mathrm{~m} / \mathrm{Q}$. Due to the lack of lonic states with M/Q greater than 10.0 , only the range of 1 to $10 \mathrm{kV}$ is plotted although the range out to $12.4 \mathrm{kV}$ is used in $\mathrm{V}$ for determining the background. Note that, with the exception of a portion of the gap at $2.5 \mathrm{kV}$, the gaps have been filled in by the shifting spectra. 


\section{APPENDIX B: $x^{2}$ FITS TO MODELS}

In order to perform a $x^{2}$ fit of the observations to predicted spectra based on equation 3 , the recombination and ionization rates must be established. The collisional ionization coefficients were calculated following the method of Summers (1974a) using the binding energies tabulated by Lotz (1968). The dominant ionization mode was assumed to be direct collisional ionization of either a valence or inner shell electron from the ground state. For some ground states $\left(2 s^{2} 2 p, 2 s^{2} 2 p^{2}, 2 p^{6} 3 s, 2 p^{6} 3 s^{2}, 3 s^{2} 3 p\right.$, and $3 s^{2} 3 p^{2}$ ) corrections for autoionization were made using the correction factors tabulated by Summers (1974a, 1974b).

The recombination coefficients were obtained by interpolation of the tables of summers $(1974 a, b)$. The processes included were radiative recombination, dielectronic recombination (with secondary autoionization), and 3-body recombination. The coefficients were calculated at a density of $10^{8} \mathrm{~cm}^{-3}$ because, for the high ionization states under consideration, there is negligible density dependence. The information provided by Summers and Lotz only allows calculations of recombination and lonization coefficients when there are less than 18 electrons on the ion. The $\mathrm{Fe}^{+5}$ to $\mathrm{Fe}^{+8}$ coefficients were obtained frow Jordan ( 1969 and private communication).

\section{a) The January 3, 1975 Event}

On January 3, 1975 a heavy Ion spectrum was obtained during a NCDE (see Fig. 1). Froa self-consistent arguments the January 3 spectrum is interpreted 
to come from a solar region with an unusually high temperature. The ratio of the $M / Q=2.29$ and 2.67 peaks, the shoulder on the $M / Q$ peak at $3.5\left(\mathrm{Fe}^{+15}\right)$, the deficit of counts at $M / Q \sim 4$, and the envelope of peaks at $M / Q>4$ all imply a hot temperature. Certainly the temperature is not as high as the one for March 5, 1973, but it is probably hotter than the April 2, 1975 and May 4, 1973 spectra (Fig. 2) because of the apparent large amount of $\mathrm{Fe}^{+15}$.

A $x^{2}$ criterion will be used to judge the quality of fit to the data using the Newkirk and CME models. In Figure 1, the brackets above each curve delineate the ranges for which the observed counts were summed together. Each bracket is designed to be influenced predominantly by a particular ion. A constant background found from the observations at $M / Q>8.6$ was subtracted from the entire spectrum, and a variance was determined (based on Poisson statistics) for each bracket. Although use of the $M / Q=2.29$ and 2.67 peaks would have increased our sensitivity over a greater ramge of radil, the peaks at $M Q Q<3$ include several different elements whose relative solar wind abundances artz only poorly known.

For giver: values of $T_{0}, a$, and $r$ the counts in each bracket were predicted by first solving equation (3) for carbon, nitrogen, oxygen, neon, magnesium, silicon, sulfur, and iron. The resulting ionization balances were folded through the known instrument response function assuming a local thermal temperature (which reproduced the shapes of the individual peaks) and abundances used in previous solar wind studies (e.g., Bame et al., 1979). The use of the instrumental response function rather than the peak height (e.g., Bame et al., 1979) was necessitated by the fact that the $\mathrm{Fe}^{+16}$ peak was not completely resolved from the $\mathrm{Fe}^{+15}$ peak and because in this presentation, the peak heights sometimes do not reflect the integrated counts in the vicinity of the peak's location. Due to the high temperature at which these spectra form, 
only silicon, sulfur, and iron significantly contribute to the bracketed ranges in Figure 1.

Figure 7 presents various cuts through the $x^{2}$ surface for the January 3 , 1975 spectrum. Figure $7 \mathrm{a}$ is for the Newkirk model and Figure 7b is for the CME model. The solid line of the upper panel shows the location of the minimum $x^{2}$ as a function of $\gamma_{0}$ and $x$ evaluated at the optimum (i.e., lowest $\left.x^{2}\right) T_{0}$. The solid line of the middle panel shows the location of the minimum $x^{2}$ as a function of $T_{0}$ and a evaluated at the optimum $\gamma_{0}$. The broken curves indicate the volume in $T_{0}, \alpha, \gamma$ space which has a $68 \%$ probability of containing the true model parameters based on the method of Lampton, Margon, and Boyer (1976). Combining the upper two panels gives an indication of the three-dimensional shape of the $x^{2}$ surface. The lower panel gives the $x^{2}$ as a function of $a$ along the bottom of the valley in the $T_{0}-\gamma x^{2}$ space.

We consider first the fit to the Newkirk density model. Here, a statistically significant minimum in the $x^{2}$ surface is observed at $x_{\text {MIN }}^{2}=$ 8.28 with 5 degrees of freedom at $T_{0}=3.4 \times 10^{6}, \alpha=0.3$, and $Y=1.0$. An inspection of the contributors to the $x^{2}$ revealed that, at all points along the $x^{2}$ minimum, $\mathrm{Fe}^{+14}$ contributed from $30 \%$ to $50 \%$ of the total $x^{2}$. The peak predicted at $\mathrm{Fe}^{+14}$ was always larger than that observed, perhaps due to uncertainties in the autoionization correction factor. If the $\mathrm{Fe}^{+14}$ peak is omitted from the $x^{2}$ calculation, the minimum $x^{2}$ becomes 5.8 with 4 degrees of freedom.

The $\alpha$ value for the best Newkirk model $\left(T_{0} \sim 3.4 \times 10^{6}, \alpha \sim 0.3\right)$ agrees well with values for a heat conduction dominated, spherically symetric corona $(a=2 / 7)$. The $a \sim 0.3$ also agrees with the average value found for the normal interstream spectra (Bame et al., 1974). In fact, the results of Figure $7 a$ are almost identical to the results for the interstream spectra 

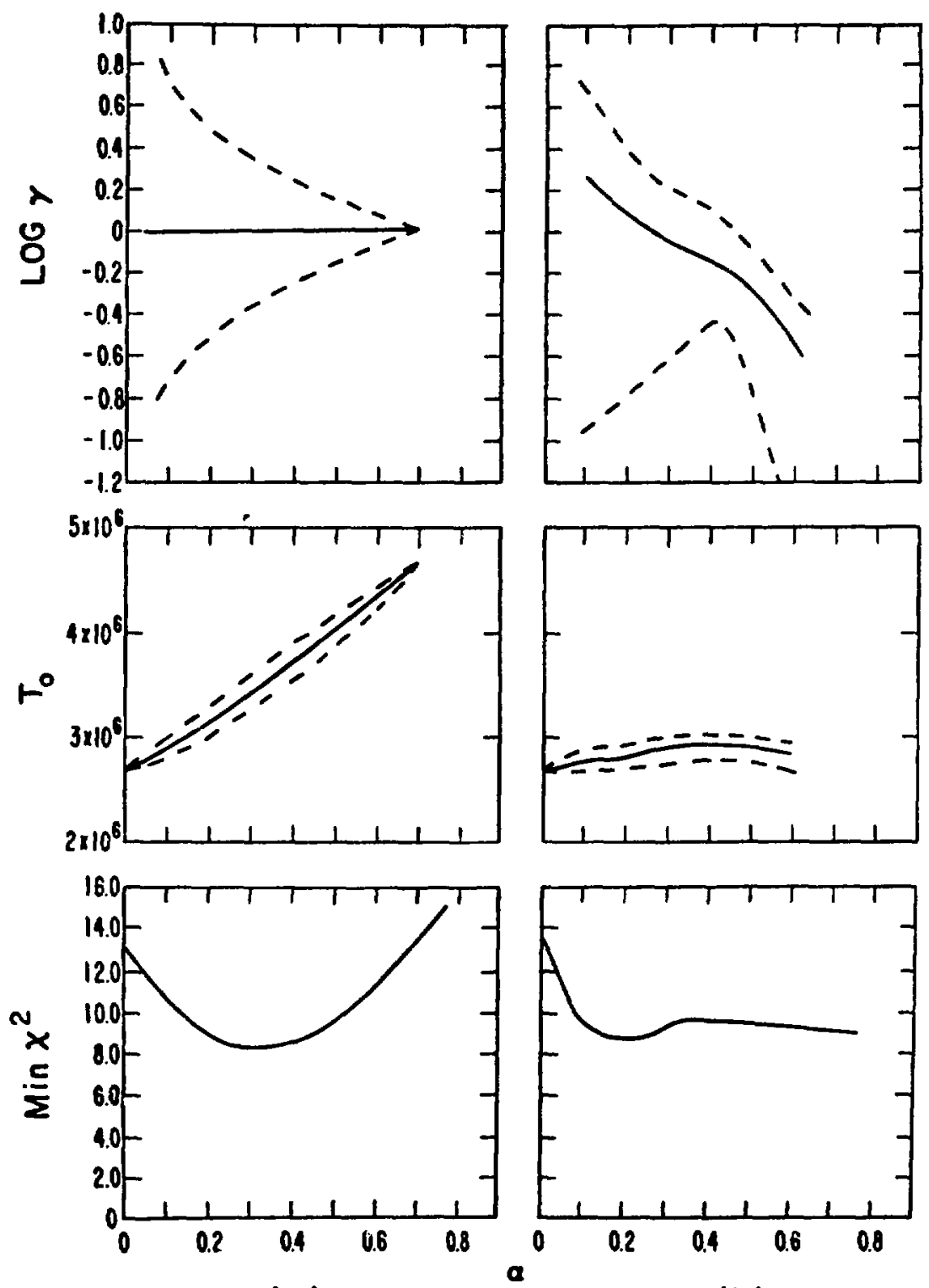

(A)

(B)

Fig. 7. $\chi^{2}$ surfaces for the January 3, 1975, spectrum (a) Newkirk model, (b) CME model. The upper two panels show the location of the $x^{2}$ minimum in $\gamma_{0}-\alpha$ space and $T_{0}-\alpha$ space. The lower panel shows the $\chi^{2}$ at the minimum as a function of $\alpha$. 
except $T_{0}$ is higher. Bame et al. found that the average $T_{0}$ for the interstream spectra is $\sim 1.84 \times 10^{6} \circ \mathrm{K}$ and the average a was 0.29 . The higher $T_{0}$ for the January 3, 1975 spectrum implies, for example, that the Iron freezes in at $\wedge$ $2.5 \times 10^{6}$ rather than the $1.58 \times 10^{6}{ }^{\circ} \mathrm{K}$ found for the interstream spectra. The ract that the best fitting value of $\gamma$ was 1.0 means that no coronal density enhancement is needed for the Newkirk model to explain the observed heavy ion spectrum. Although a factor of 4 or more increase in density can not be ruled out by the data, we conclude that the Newkirk model does not necessitate a coronal density enhancement to explain this NCDE. The spectrum could have arisen from a corona whose only difference from the interstream models is a higher $\mathrm{T}_{0}$.

An inspection of Fig. $7 \mathrm{~b}$ shows that the CME model can fit the January 3 , 1975 spectrum almost as well as the Newkirk model. Here, the minimum $x^{2}$ occurred at a $\sim 0.2$ and was equal to 8.7 . This value of $x^{2}$ is only 58 larger than the that for the Newkirk model. At the $x^{2}$ minimum $\gamma$ is 1.0 , which is equivalent to a density enhancement approximately that of the September 14-15, 1973 CME event. Thus the same data are consistent with both a normal density and a density enhanced coronal model.

In the January 3, 1975 spectrum, the heavy ion distribution was close to equilibrium and could be fit by both the Newkirk and CME models. In the CME models the freezing-in occurred low in the atmosphere $\left(<1.5 \mathrm{R}_{0}\right)$ where the density gradient from equation 4 was sufficiently steep that the various ions from a particular element (e.g., iron) freeze-in within a small radial distance. Thus, the CME model need not always give extreme, nonequilibrium distributions. 


\section{b) The March 5,1973 Event}

In contrast to the January 3, $1975 \mathrm{NCDE}$, the March 5, $1973 \mathrm{NCDE}$ had an extreme, nonequilibrium ionization distribution (see Fig. 1). Following the same procedures as outlined above, the minimum $x^{2}$ found for the March 5,1973 spectrum using both the Newkirk and CME models was $\sim 22$ (with 5 degrees of freedom). Such a large value of $x^{2}$ occurred because the variances on the observed counts were small (standard deviation to mean ratios of $\backsim .02$ ). In this case small errors in the model or rate coefficients would tend to dominate the $x^{2}$ calculation. For example, the quoted accuracy of the recombination and ionization rate coefficients ( $s$ factor of 2 ) are sufficient to cause errors in the ionization balance which would dominate the $x^{2}$ calculation. Effectively the $x^{2}$ always had a minimum when the model fit the $\mathrm{Fe}^{+16}$ and $\mathrm{Fe}^{+15}$ peaks (which had the smallest statistical uncertainities) and was nearly independent of the data at $M / Q$ values greater than $4 . A x^{2}$ value of 22 corresponded to fitting the $\mathrm{Fe}^{+16}$ and $\mathrm{Fe}^{+15}$ peaks exactly and predicting approximately zero counts for the rest of the spectrum. The January 3, 1975 spectrum did not have this problem because its peaks were all about the same height and thus they all had roughly the same variances.

In order to be less sensitive to the uncertainties in the recombination and ionization coefficients we will evaluate which model best fits the envelope of peaks in the March 5, 1973, spectrum. Instead of using the variances derived from the Poisson counting statistics, each bracket was assigned a variance such that the ratio of the standard deviation to the counts were equal. Such a formulation gives a minimum $x^{2}$ for the model which best fits the envelope of peaks in the observations because a difference between an observation and predicted value for a peak will contribute the same to the $x^{2}$ for all peaks if the fractional error is the same. 
Figure 8 shows various cuts through the modified $x^{2}$ surface (in arbitrary units) for the March 5, 1973 spectrum. The solid lines in the upper two panels show where the minimum $x^{2}$ occurred as a function of $T_{0}$ and a for two different values of $\gamma$. The lower panels show the value of the minimum $x^{2}$ as a function of $\alpha$. The broken and dotted lines in the upper panel are respectively the 3.0 and 3.5 contours in $T_{0}$, a space. These contours are meant to indicate the general shape of the $x^{2}$ surface and are not confidence levels.

Figure $8 \mathrm{a}$ is based on the Newkirk model. The $x^{2}$ surface had two troughs intersecting the $T_{0}$ axis at $2.0 \times 10^{6}$ and $3.6 \times 10^{6 \circ} \mathrm{K}$. The trough beginning at $3.6 \times 10^{60} \mathrm{~K}$ provided a better fit to the $\mathrm{Fe}^{+15}$ and $\mathrm{Fe}^{+16}$ peaks and the $2 \times$ $10^{60} \mathrm{~K}$ trough tended to fit the $\mathrm{Fe}^{+13}$ to $\mathrm{Fe}^{+9}$ peaks. It is seen that the $\mathrm{fit}$ for the Newkirk model is very poor unless a is greater than $\sim 1.0$. Such a high value of a is difficult to justify if the atmosphere is dominated by heat conduction. However, heat conduction may not be important if the magnetic field has a closed loop structure so that $a \geqslant 1$ may be physically acceptable. Figure $8 \mathrm{~b}$ presents the modified $x^{2}$ for the CME models. Not only is the CME $x^{2}$ smaller, but the CME obtains its best fit ( $\alpha \sim 0.43, \mathrm{~T}_{0} \sim 4.3 \times 10^{6}{ }^{\circ} \mathrm{K}$ ) without resorting to a large $\alpha$.

The best fit model predicts ionization state densities which differ systematically from the observations. The $\mathrm{Fe}^{+16}$ and $\mathrm{Fe}^{+15}$ peaks contribute approximately $50 \%$ of the $x^{2}$ because the model predicts a smaller peak at $M / Q=$ 3.5 than observed. If Indeed the March 51975 spectrum originated from a CME, there are ample reasons why the observations might differ from the CME model. 1) Perhaps the areal expansion was not symmetric about a point on the surface of the sun. Usuall* only the center of the loops in a CME are symmetric about a point near the surface. The plasma sampled at 1 AU could have come from the 


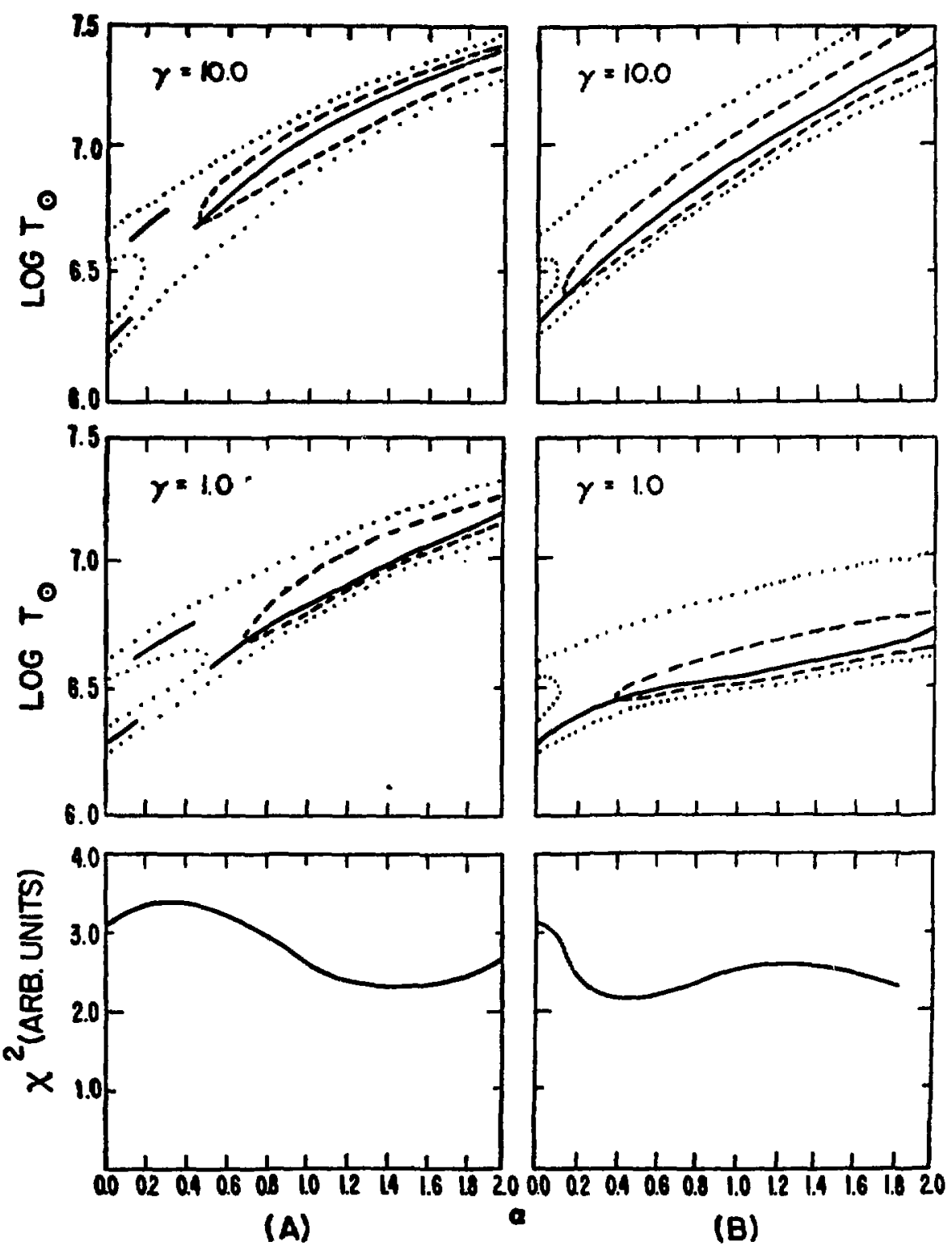

Fig. 8. Modified $\chi^{2}$ surfaces for the March 5, 1973, spectrum (a) Newkirk model, (b) CME model. 
edge of a CME which often appears like a streamer. 2) The velocity for the March event may not have been constant. 3) The density profile may have been very different from that of the September 14-15, 1973, CME in which equation 4 was based. 4) The event responsible for the March spectrum could have been very complex in which the assumed temperature structure (Eq. 1) did not hold.

What can be learned from the March 5, 1973 spectrum and the models we have fit to it? The above-mentioned uncertainties prevent quantitative results. However, several qualitative results are possible. 1) The March 5 spectrum is indeed from an anomalously high-temperature corona. 2) The Newkirk model can not fit the data without resorting to large values of a (see Fig. 8a). Thus the spectrum indeed has an extreme, nonequilibrium ionization distribution. Another possible explanation of the nonequilibrium distribution is that the solar wind contained a mixture of two plasmas (see also Bame et al., 1973). However, if the CME interpretation is that a loop of material expands away from the sun, such a loop structure would tend to restrict double streaming and mixture because the material would then have to cross field lines. 
I wish to thank all those who made this thesis possible. In particular, I wish to thank everyone in Group P-4 of the Los Alamos Scientific Laboratory who not only gave me an enfoyable and stimulating place to work, but also built, flew, and processed the Vela and Imp instruments upon which this work was based. This includes Samue1 Bame, John Asbridge, Harry Felthauser, Georgia Hall, J. Paul Glore, and Burt McCloud for the design and calibration of the instruments; and Ear1 Tech, Marilyn Halbig, Wynoka Miller, Dorothy Smith, Patricia Max, Sally Duran, Rose Watts, Mary Warren, and Jean Dabni:y for the data processing. Jean DeField deserves a special thanks for typing innumerable versions.

I a1so thank the US Department of Defense and the US Department of Energy who jointly sponsor the Vela program and the US Nationa1 Aeronautics and Space Administration who sponsored the Imp program. In addition, this work was supported in part by NASA grant S57094A and was performed under the auspices of the US Department of Energy.

Samuel Bame, Jack Gosling, and espectally William Feldman contributed by many helpful discussions and useful comments on the manuscript. Richard Blake deserves special gratitude for his many years of advice and support as my thesis advisor. 
REFERENCES

Asbridge, J. R., Bame, S. J., and Strong, I. B. 1968, J.Geophys. Res., 73, 5777 .

Bame, S. J. 1972, in Solar Wind, ed. C. Sonett, P. Coleman, Jr., and J. W1lcox (NASA SP-308; Washington: Government Printing Office), p. 535.

Bame, S. J., Hundhausen, A. J., Asbridge, J. R., and Strong, I. B. 1968, Phys. Rev. Letters, 20, 393.

Bame, S, J., Asbridge, J. R., Hundhausen, A. J., and Montgomery, M. D. 1970, J. Geophys. Res., 75, 6360.

Bame, S. J., Asbridge, J. R., Feldman, H. C., and Kearney, P. D. 1973, Am. Geophys. Union EQS, 24,438 (abstract). 1974, Solar Phys., 35, 137.

Bame, S. J., Asbridge, J. R., Feldman, W. C., Montgomery, M. D., and Kearney, P. D. 1975, Solar Phys, 43, 463.

Bame, S. J., Asbridge, J. R., Feldman, W. C., Fenimore, E. E. and Gosling, J. T. 1979, Solar Phys., 62, 179.

Belcher, J. W. and Davis, L., Jr. 1971, J. Geohys. Res., J6, 3534.

Dryer, M., Steinolfson, R. S., and Wu, S. T. 1976, Am. Geophys. Union EOS, 57, 998 (abstract).

Dulk, G. A., Smerd, S. F., MacQueen, R. M., Gosling, J. T., Magun, A., Stewart, R. T., Sheridan, K. V., Robinson, R. D., and Jacques, S. 1976, Solar Phys., 42, 369 .

Feldman, W. C., Asbridge, J. R., Bame, S. J., and Kearney, P. D. 1974, J. Geophys, Res., 79, 1808. 
Feldman, W. C., Asbridge, J. R., Bame, S. J., and Gosling, J. T. 1978, Je Geophys. Res, 83, 2177.

Ge1ss, J. Eberhardt, P., Buhler, F., and Me1ster, J. 1970, J.Geophys. Res., I5, 5972.

Gosling, J. T., Pizzo, V., and Bame, S. J. 1973, J. Geophys, Res, 78, 2001. Gosling, J. T., Hildner, E., MecQueen, R. M., Munro, R. H., Poland, A. I., and Rast, C. L. 1974, J. Geophys. Res,, 79, 4581. Gosling, J. T., Hildner, E., Asbridge, J. R., Bame, S. J., and Feldman, W. C. 1977 , J. Geophys. Res,, 82, 5005.

Hirshberg, J., Alksne, A., Colburn, D. S., Bame, S. J., and Hundhausen, A. J. 1970, J. Geophys, Res., 75, 1.

Hirshberg, J., Bame, S. J., and Robbins, D. E. 1972, Solar Phis., 23, 467. Holzer, T. E. and Axford, W. I. 1970, J. Geophys, Res, 75, 6354. Hundhausen, A. J. 1972 in Solar Wind, ed. C. Sonett, P. Coleman, Jr., and J. Wilcox (NASA SP-308, Washington: Government Printing office), p. 393. Hundhausen, A. J., Gilbert, H. E., and Bame, S. J. 1968, J.Geophys. Res., 73, 5485.

Jordan, C. 1969, M.N.R.A.S., 142, 501.

Lampton, M., Margon, B., and Boyer, S. 1976, AD._., 208, 177.

Lange, T., and Scherb, R., 1970, J.Geophys, Res., 75, 6350.

Levine, R. H. , 1978, J. Geophys, Rese, 83, 4193.

Lotz, W. 1968, J.O.S.A., 58, 915.

MacQueen, R. M., J. A. Eddy, J. T. Gosling, E. Hildner, R. H. Munro, G. A

Newkirk, A. I. Poland, and C. L. Ross, 1974, ARe J., 187, L85.

Montgomery, M. U., Asbridge, J. R., Bame, S. J., and Feldman, W. C. 1974, J.

Geophys. Res., 79, 3103.

Newkirk, G., Jr. 1967, Ann. Rer. Astron, and Astroohys., 5, 213.

Pizzo, V. 1976, Ph.D. thesis, University of Colorado. 
Pneuman, G. W., 1973, Solar Phys,, 28, 247.

Robbins, D. E., Hundhausen, A. J., and Bame, S. J. 1970, J. Geophys. Res., 75, 1178.

Summers, H. P. 1974 a, Apoleton Laboratory Internal Report, 367. 1974b, M.N.R.A.S, 169, 663. 